\title{
Azimuthal structures in the wind and chromosphere of the Herbig Ae star AB Aurigae.
}

\section{Results from the MUSICOS 1992 campaign*}

T. Böhm ${ }^{1,2}$, C. Catala ${ }^{3}$, J.-F. Donati ${ }^{3}$, A. Welty ${ }^{\dagger, 4}$, J. Baudrand ${ }^{2}$, C.J. Butler ${ }^{5}$, B. Carter ${ }^{6}$, A. Collier-

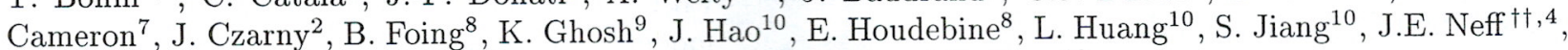
D. Rees ${ }^{11}$, M. Semel ${ }^{2}$, T. Simon ${ }^{12}$, A. Talavera ${ }^{13}$, D. Zhai ${ }^{10}$ and F. Zhao ${ }^{10}$

1 European Southern Observatory, Karl-Schwarzschildstr. 2, 85748 Garching, Germany internet: tboehm@eso.org

2 Département d'Astrophysique Extragalactique et de Cosmologie et Unité de Recherche associée au CNRS 173 et à l'Université Paris 7, Observatoire de Paris, Section de Meudon, 92195 Meudon Principal Cedex, France

3 Laboratoire d'Astrophysique et Unité de Recherche associée au CNRS 285, Observatoire Midi-Pyrénées, France

4 Pennsylvania State University, Department of Astronomy and Astrophysics, 525 Davey Laboratory, University Park, PA 16802, U.S.A.

5 Armagh Observatory, Armagh BT61 9DG, N. Ireland, UK

${ }^{6}$ University of New South Wales, Australia

7 School of Physics \& Astronomy, University of St Andrews, North Haugh, St Andrews, Fife, Scotland KY16 9SS

8 Solar System Division, ESA Space Science Department, ESTEC (SO), postbus 299, NL-2200 AG, Noordwijk, The Netherlands

9 Indian Institute of Astrophysics, Vainu Bappu Observatory, Kavalur, Alangayam 635701, Tamilnadu, India

10 Beijing Astronomical Observatory, China

11 CSIRO Division of Radiophysics, Epping, NSW 2121, Australia

12 Institute for Astronomy, University of Hawaii, 2680 Woodlawn Drive, Honolulu HI 96822, U.S.A.

13 LAEFF/INTA, P.O. Box 50727, 28080 Madrid, Spain

Received February 22; accepted May 2, 1996

\begin{abstract}
The observations of the MUSICOS 1992 campaign concerned three scientific programs, among which was the study of azimuthal structures in the wind and chromosphere of the pre-main sequence Herbig Ae star $\mathrm{AB}$ Aur. The HeI5876 $\AA$ line of AB Aur, which is formed in the expanding chromosphere of this star, most probably in the innermost parts of its wind, was continuously monitored at a spectral resolution of 30000 for about 4 days. The line was discovered to be variable in a spectacular way, the profile changing from pure emission to a composite profile including a deep absorption component in the course of a few hours. In this paper we present strong clues that suggest a rotational modulation of the line, with a period corresponding to the stars rotation period of 32 hrs. We confirmed the covariation of several non-photospheric spectral lines, formed at very different radial distances of the star, which strengthens the idea that the variability is the signature of azimuthal structures in the wind of $A B$ Aur, most probably due to magnetically confined streams or loops emanating from the stellar surface. We present the data collected during the MUSICOS 1992 campaign, but also a data set of the HeID3 line obtained with FOE at KPNO during the years 1991-1994 and a series of IUE spectra containing the Mg II h \& $\mathrm{k}$ UV lines obtained one month prior to the campaign. A thorough discussion of the possible interpretations of the spectacular variations of the HeI $5876 \AA$ line is presented.
\end{abstract}

Key words: line: formation — line: profiles — stars: magnetic fields — stars: pre-main sequence — stars: AB Aur - stars: emission line, Be

Send offprint requests to: T. Böhm (German address)

* Based on observations obtained during the MUSICOS 92

MUlti-SIte COntinuous Spectroscopic campaign from the
National Solar Observatory McMath-Pierce $1.5 \mathrm{~m}$ telescope, University of Hawaii $2.2 \mathrm{~m}$, Beijing Observatory Xinglong $2.16 \mathrm{~m}$, La Palma $4.2 \mathrm{~m}$ William Herschel Telescope, and 


\section{Introduction}

The Herbig Ae/Be stars are pre-main sequence objects of intermediate mass $\left(2-5 M_{\odot}\right)$. They show conspicuous signs of intense activity, extended chromospheres and strong stellar winds, with mass loss rates in the range of $10^{-9}-10^{-6} M_{\odot} \mathrm{yr}^{-1}$. In the case of the prototype Herbig Ae star AB Aur (spectral type A0e, $T_{\text {eff }}=$ $10000 \mathrm{~K}$ ), a semi-empirical model of its wind and chromosphere (Catala \& Kunasz 1987) led to an estimate of its mass loss rate $\left(10^{-8} M_{\odot} \mathrm{yr}^{-1}\right)$ and to the determination of the structure of its chromosphere (maximum temperature $\approx 17000 \mathrm{~K}$; size $\approx 1.5 R_{*}$ ).

The location of the Herbig Ae/Be stars in the HR diagram indicates that they are in the radiative phase of their contraction towards the main sequence, i.e., that they do not possess outer convective zones, according to the standard theory of stellar evolution (Iben 1965; Gilliland 1986). Considering that stellar activity is generally attributed to magnetic fields and/or acoustic waves generated in a convection zone, these active phenomena are quite paradoxical in the Herbig Ae/Be stars.

Nevertheless, we already have some indirect clues that the activity of the Herbig Ae stars might be of magnetic origin. Praderie et al. (1986) and Catala et al. (1986) observed rotational modulation of the $\mathrm{Mg}$ II and Ca II resonance lines in the spectra of the prototype star AB Aur (spectral type A0e, $T_{\text {eff }}=10000 \mathrm{~K}$ ), with a period consistent with the stellar rotation $\left(P_{\text {rot }} \simeq 32 \mathrm{hrs}\right)$. By analogy with the solar wind, they proposed that the modulation might be due to the corotation of fast and slow streams, the wind structure being controlled by a surface magnetic field.

However, the link between the stellar wind modulation and a structured magnetic field is far from being firmly established. In particular, we have no clear indication that the source of the modulation is near the stellar surface, since the lines for which such a modulation has been observed are formed far out in the wind. Are we seeing a corotating structure linked to the stellar surface by a magnetic field, or some other type of variable phenomenon occuring in the wind itself, like e.g., inhomogeneities passing periodically through the line of sight?

Observatoire de Haute-Provence $1.52 \mathrm{~m}$ telescopes, and based on

observations by the International Ultraviolet Explorer (IUE) collected at the Goddard Space Flight Center (NASA) and the Villafranca Satellite Station (ESA). † Visiting Astronomer, Kitt Peak National Observatory, National Optical Astronomy Observatories, which is operated by the Association of Universities for Research in Astronomy, Inc. (AURA), under cooperative agreement with the National Science Foundation. If Visiting Astronomer, National Solar Observatory, National Optical Astronomy Observatories, which is operated by the Association of Universities for Research in Astronomy, Inc. (AURA), under cooperative agreement with the National Science Foundation.
In this framework, the study of a variable spectral line formed close to the stellar surface is of major interest; the strongly variable HeI $5876 \AA$ line, which is formed at the very base of the wind/chromosphere complex (Catala et al. 1993), is an excellent variability tracer. The simultaneous variation of this line and the Fe II $5018 \AA$ line (formed in the bulk of the expanding chromosphere) indicates the presence of a globally structured chromosphere (Böhm \& Catala 1993a; Catala et al. 1993). However, due to the lack of a consistent data set, these authors were only able to conclude that the observations are in good agreement with a rotational modulation, which does not prove the model.

The goal of the "AB Aur" program of the MUSICOS 1992 campaign was therefore to observe the HeI5876 $\AA$ line during several stellar rotation periods with a complete time coverage to verify whether the rotational modulation is also found close to the stellar surface, and to constrain better the azimuthal structure of the wind and chromosphere by time resolved observations. The HeI $5876 \AA$ line was monitored at a resolution of 30000 nearly continuously for about 4 days. During the preliminary analysis of the observations a spectacular variabilty of this line was discovered (Catala et al. 1994), the profile changing from pure emission to a composite profile including a deep absorption component in the course of a few hours. It has been suggested that this variability can be the signature of azimuthal structures in the wind of $A B$ Aur.

This paper presents a thorough description and analysis of the MUSICOS 1992 observations, but includes also a large data set of medium resolution observations ( $R \simeq 12000$ ) of the same line obtained during 1991, 1992, 1993 and 1994 with the Penn State Fiber Optic Echelle (FOE) at Kitt Peak National Observatory (KPNO), as well as a data set of the $\mathrm{MgIIh}$ and $\mathrm{k}$ UV lines observed by IUE one month prior to the campaign $(R \simeq 15000)$.

Section 2 presents the observations and data reductions. Section 3 gives the results of the observed variability. In Sect. 4 the results are discussed and conclusions are drawn in Sect. 5.

\section{Observations and data reduction}

This section describes in some detail the instrumentation used for the MUSICOS 1992 campaign, but also for the FOE/KPNO and IUE observations included in the present analysis. Table 1 shows the participating sites and the corresponding observers. Some additional spectra were obtained at Vainu Bappu Observatory, India (observer K.K. Ghosh) during the MUSICOS 1992 campaign; due to their low spectral resolution and low $\mathrm{S} / \mathrm{N}$ ratios they could not be included in the analysis of this article. 
Table 1. Instruments - Observers. (E): echelle spectrograph. From the KPNO/FOE echelle spectrograph, only He ID3 line data are presented in this paper. Instruments/observers participating in the MUSICOS 1992 campaign are described in the first group; additional data are provided by instruments/observers of the second group

\begin{tabular}{|c|c|}
\hline $\begin{array}{l}\text { UH } 2.2 \mathrm{~m}+\text { MUSICOS (E) } \\
\text { OHP } 1.52 \mathrm{~m}+\text { Aurélie } \\
\text { Xinglong } 2.16 \mathrm{~m}+\text { ISIS } \\
\text { Kitt Peak } 1.5 \mathrm{~m} \text { McMath-Pierce } \\
\text { WHT } 4.2 \mathrm{~m}+\text { Utrecht }(\mathrm{E})\end{array}$ & $\begin{array}{l}\text { T. Böhm, J. Baudrand, J. Czarny, T. Simon } \\
\text { C. Catala, J.-F. Donati } \\
\text { S. Jiang, J. Hao, L. Huang, D. Zhai, F. Zhao, B. Foing } \\
\text { J.E. Neff } \\
\text { E. Houdebine }\end{array}$ \\
\hline $\begin{array}{l}\text { KPNO/FOE } 2.1 \mathrm{~m} \text { and coudé feed }[(\mathrm{E})] \\
\text { IUE }\end{array}$ & $\begin{array}{l}\text { A. Welty, J. Hall } \\
\text { T. Simon, A. Talavera }\end{array}$ \\
\hline
\end{tabular}

\subsection{Data from the MUSICOS 1992 campaign}

This section describes in some detail the instrumentation used during this campaign, and the methods of data reduction employed. All data are accessible via anonymous ftp at "mus.obs-mip.fr", directory "pub/mus92".

Because the instruments were not identical from site to site, different procedures were used for the various sites. The log of the observations is given in Table 2; included are also 2 additional spectra from Xinglong and 3 additional spectra from UH, obtained within the 7 days following the AB Aur observations of the MUSICOS 1992 campaign.

\subsubsection{UH $2.2 \mathrm{~m}$}

The MUSICOS fiber-fed echelle spectrograph, described in Baudrand \& Böhm (1992), was mounted on the 2.2 $\mathrm{m}$ telescope of the University of Hawaii, at Mauna Kea Observatory. The detector was a $2048 \times 2048$ Tektronix CCD.

The data were reduced using dedicated software, developed by one of us (JFD), which is based on the optimal extraction algorithm (Horne 1986) and uses a 2D polynomial fit of $\mathrm{Th} / \mathrm{Ar}$ line spectra to calibrate in wavelength.

Low amplitude fringes appearing both on the stellar and flat-field spectra could not be entirely removed. This problem does not affect the analysis of line profile variations, which have a much higher amplitude than the fringes.

\subsubsection{OHP $1.52 \mathrm{~m}$}

We used the Aurélie spectrograph on the $1.52 \mathrm{~m}$ telescope of the Observatoire de Haute Provence. This instrument was equipped with a Thomson 1D 2048 pixel detector.

The OHP data, as well as the Xinglong and Kitt Peak data, were reduced with the eVe reduction software developed at Paris-Meudon Observatory.

After subtraction of an average of 10 electronic bias frames obtained before and after the stellar exposure, the stellar spectra were divided by an average of 10 flatfield spectra. They were then calibrated using Th/Ar lamp spectra, cleaned from cosmic ray hits, and normalized to the continuum.

\subsubsection{Xinglong $2.16 \mathrm{~m}$}

The fiber-fed ISIS spectrograph (Felenbok \& Guérin 1988) was mounted on the $2.16 \mathrm{~m}$ telescope of the Beijing Astronomical Observatory, with a $512 \times 512$ Tektronix CCD. A special improvement of this instrument, consisting of the installation of an image slicer, was performed at Meudon Observatory before shipment of the instrument to China.

As a first step of the reduction procedure, a $2 \mathrm{D}$ representation of the background, estimated from areas of the CCD free of stellar light, was subtracted from the stellar and the flatfield spectra. Cosmic ray hits were then removed by interpolating between adjacent pixels. Stellar and flatfield spectra were extracted, by simply summing the columns belonging to slices of the image slicer. The stellar spectra were subsequently divided by the flat-field spectra. This division after spectrum extraction is justified here because the flat-field spectra fall exactly on the same pixels as the stellar spectra, and, due to the fiber-feed, have the same shape perpendicularly to the dispersion. Finally, the stellar spectra were calibrated with the help of Th/Ar lamp spectra.

\subsubsection{Kitt Peak McMath-Pierce}

We used the stellar spectrograph, with a $800 \times 800 \mathrm{TI}$ CCD. Due to the image slicer used with this instrument, the flat-field spectra are wider than the stellar spectra. We nevertheless used a procedure identical to that of the Xinglong data. However, we reduced one of the spectra with a different and in principle more appropriate method: (1) background subtraction; (2) pixel-by-pixel division by the flat-field; (3) extraction and weighted average of the 
Table 2. Log of the MUSICOS 92 observations. Some additional spectra were obtained shortly after the campaign (\# 90-94). Columns: (1) \# of spectrum. (2) UT date and (3) time of observations. (4) Observing site; XL: Xinglong $2.16 \mathrm{~m}+$ ISIS spectrograph. UH: UH $2.2 \mathrm{~m}+$ MUSICOS spectrograph. KP: Kitt Peak McMath-Pierce + stellar spectrograph. WHT: WHT $4.2 \mathrm{~m}$ telescope + Utrecht Echelle spectrograph. OHP: OHP $1.52 \mathrm{~m}+$ Aurelie spectrograph. (5) Exposure time. (6) Time difference to the first spectrum. ( $\left.\Delta t_{\text {mid.exp. }}\right)$

\begin{tabular}{|c|c|c|c|c|c|c|c|c|c|c|c|}
\hline $\begin{array}{r}\# \\
(1) \\
\end{array}$ & $\begin{array}{r}\text { UT: date } \\
\text { (2) }\end{array}$ & $\begin{array}{c}\text { UT: time } \\
\text { (mid.exp) } \\
(3)\end{array}$ & $\begin{array}{l}\text { Site } \\
(4) \\
\end{array}$ & $\begin{array}{r}t_{\exp } \\
(\min ) \\
(5) \\
\end{array}$ & $\begin{array}{r}\Delta t \\
(\mathrm{hrs}) \\
(6) \\
\end{array}$ & $\begin{array}{l}\# \\
(1)\end{array}$ & $\begin{array}{r}\text { UT: date } \\
(2)\end{array}$ & $\begin{array}{c}\text { UT: time } \\
\text { (mid.exp) } \\
(3)\end{array}$ & $\begin{array}{l}\text { Site } \\
(4) \\
\end{array}$ & $\begin{array}{r}t_{\exp } \\
(\min ) \\
(5) \\
\end{array}$ & $\begin{array}{r}\Delta t \\
\text { (hrs) } \\
(6)\end{array}$ \\
\hline 1 & 3 Dec. 92 & 1403 & $X L$ & 90 & 0.00 & 46 & 7 Dec. 92 & 0255 & $\mathrm{KP}$ & 60 & 84.87 \\
\hline 2 & & 1723 & $\mathrm{XL}$ & 90 & 3.33 & 47 & & 0358 & KP & 60 & 85.92 \\
\hline 3 & & 1858 & $\mathrm{XL}$ & 90 & 4.92 & 48 & & 0459 & KP & 60 & 86.93 \\
\hline 4 & & 2000 & $\mathrm{OHP}$ & 60 & 5.95 & 49 & & 0602 & KP & 60 & 87.98 \\
\hline 5 & & 2030 & $\mathrm{XL}$ & 90 & 6.45 & 50 & & 0652 & $\mathrm{UH}$ & 30 & 88.82 \\
\hline 6 & & 2105 & OHP & 60 & 7.03 & 51 & & 0703 & $\mathrm{KP}$ & 60 & 89.00 \\
\hline 7 & & 2215 & OHP & 60 & 8.20 & 52 & & 0803 & $\mathrm{UH}$ & 30 & 90.00 \\
\hline 8 & & 2322 & OHP & 60 & 9.32 & 53 & & 0804 & $\mathrm{KP}$ & 60 & 90.01 \\
\hline 9 & 4 Dec. 92 & 0117 & $\mathrm{OHP}$ & 60 & 11.23 & 54 & & 0838 & $\mathrm{UH}$ & 30 & 90.58 \\
\hline 10 & 5 Dec. 92 & 1855 & $\mathrm{OHP}$ & 90 & 52.89 & 55 & & 0911 & $\mathrm{UH}$ & 30 & 91.13 \\
\hline 11 & & 2016 & OHP & 60 & 54.22 & 56 & & 0915 & $\mathrm{KP}$ & 60 & 91.20 \\
\hline 12 & & 2134 & OHP & 60 & 55.52 & 57 & & 0945 & UH & 30 & 91.70 \\
\hline 13 & & 2237 & OHP & 60 & 56.57 & 58 & & 1017 & $\mathrm{KP}$ & 60 & 92.23 \\
\hline 14 & & 2348 & OHP & 60 & 57.75 & 59 & & 1021 & $\mathrm{UH}$ & 30 & 92.30 \\
\hline 15 & 6 Dec. 92 & 0202 & OHP & 60 & 59.98 & 60 & & 1054 & $\mathrm{UH}$ & 30 & 92.85 \\
\hline 16 & & 0303 & OHP & 60 & 61.00 & 61 & & 1117 & $\mathrm{KP}$ & 60 & 93.23 \\
\hline 17 & & 0415 & OHP & 60 & 62.20 & 62 & & 1130 & $\mathrm{UH}$ & 30 & 93.45 \\
\hline 18 & & 0513 & OHP & 60 & 63.17 & 63 & & 1211 & $\mathrm{UH}$ & 45 & 94.13 \\
\hline 19 & & 0619 & UH & 30 & 64.27 & 64 & & 1300 & $\mathrm{UH}$ & 45 & 94.95 \\
\hline 20 & & $07 \quad 10$ & $\mathrm{UH}$ & 60 & 65.12 & 65 & & 1353 & UH & 45 & 95.83 \\
\hline 21 & & 0759 & $\mathrm{UH}$ & 30 & 65.93 & 66 & & 2329 & WHT & 10 & 105.43 \\
\hline 22 & & 0831 & $\mathrm{KP}$ & 42 & 66.47 & 67 & & 2340 & WHT & 10 & 105.62 \\
\hline 23 & & 0834 & $\mathrm{UH}$ & 30 & 66.52 & 68 & & 2356 & WHT & 10 & 105.89 \\
\hline 24 & & 0920 & $\mathrm{UH}$ & 30 & 67.28 & 69 & 8 Dec. 92 & 0009 & WHT & 10 & 106.10 \\
\hline 25 & & 0926 & $\mathrm{KP}$ & 60 & 67.38 & 70 & & 0023 & WHT & 10 & 106.33 \\
\hline 26 & & 0955 & UH & 30 & 67.87 & 71 & & 0038 & WHT & 10 & 106.58 \\
\hline 27 & & 1032 & $\mathrm{KP}$ & 60 & 68.48 & 72 & & 0049 & WHT & 10 & 106.77 \\
\hline 28 & & 1035 & $\mathrm{UH}$ & 30 & 68.53 & 73 & & 0102 & WHT & 10 & 106.98 \\
\hline 29 & & 1103 & $\mathrm{UH}$ & 15 & 69.00 & 74 & & 0119 & WHT & 10 & 107.27 \\
\hline 30 & & 1123 & $\mathrm{UH}$ & 18 & 69.33 & 75 & & 0131 & WHT & 10 & 107.47 \\
\hline 31 & & 1133 & $\mathrm{KP}$ & 60 & 69.50 & 76 & & 0143 & WHT & 10 & 107.67 \\
\hline 32 & & 1158 & $\mathrm{UH}$ & 18 & 69.91 & 77 & & 0155 & WHT & 10 & 107.87 \\
\hline 33 & & 1222 & $\mathrm{UH}$ & 18 & 70.31 & 78 & & 0207 & WHT & 10 & 108.07 \\
\hline 34 & & 1247 & $\mathrm{UH}$ & 18 & 70.73 & 79 & & $02 \quad 19$ & WHT & 10 & 108.27 \\
\hline 35 & & 1311 & $\mathrm{UH}$ & 18 & 71.13 & 80 & & 0231 & WHT & 10 & 108.47 \\
\hline 36 & & 1334 & UH & 18 & 71.52 & 81 & & 0334 & WHT & 10 & 109.52 \\
\hline 37 & & 1407 & $\mathrm{UH}$ & 18 & 72.07 & 82 & & 0345 & WHT & 10 & 109.70 \\
\hline 38 & & 1415 & $\mathrm{XL}$ & 90 & 72.20 & 83 & & 0402 & WHT & 10 & 109.98 \\
\hline 39 & & 1430 & $\mathrm{UH}$ & 18 & 72.45 & 84 & & 0414 & WHT & 10 & 110.18 \\
\hline 40 & & 1605 & $\mathrm{XL}$ & 90 & 74.03 & 85 & & 0427 & WHT & 10 & 110.40 \\
\hline 41 & & 1822 & $\mathrm{XL}$ & 120 & 76.32 & 86 & & 0441 & WHT & 10 & 110.63 \\
\hline 42 & & 1851 & OHP & 60 & 76.80 & 87 & & 0453 & WHT & 10 & 110.83 \\
\hline 43 & & 1954 & $\mathrm{OHP}$ & 60 & 77.85 & 88 & & 0510 & WHT & 10 & 111.12 \\
\hline 44 & & 2127 & OHP & 60 & 79.40 & 89 & & 0521 & WHT & 10 & 111.30 \\
\hline \multirow[t]{6}{*}{45} & & 2240 & OHP & 60 & 80.62 & & & & & & \\
\hline & & & & & & 90 & 8 Dec. 92 & 2014 & $\mathrm{XL}$ & 60 & 126.18 \\
\hline & & & & & & 91 & 10 Dec. 92 & 2034 & XL & 60 & 174.51 \\
\hline & & & & & & 92 & 14 Dec. 92 & 0808 & UH & 45 & 258.08 \\
\hline & & & & & & 93 & 15 Dec. 92 & 0548 & $\mathrm{UH}$ & 20 & 279.75 \\
\hline & & & & & & 94 & & 1100 & UH & 25 & 284.95 \\
\hline
\end{tabular}




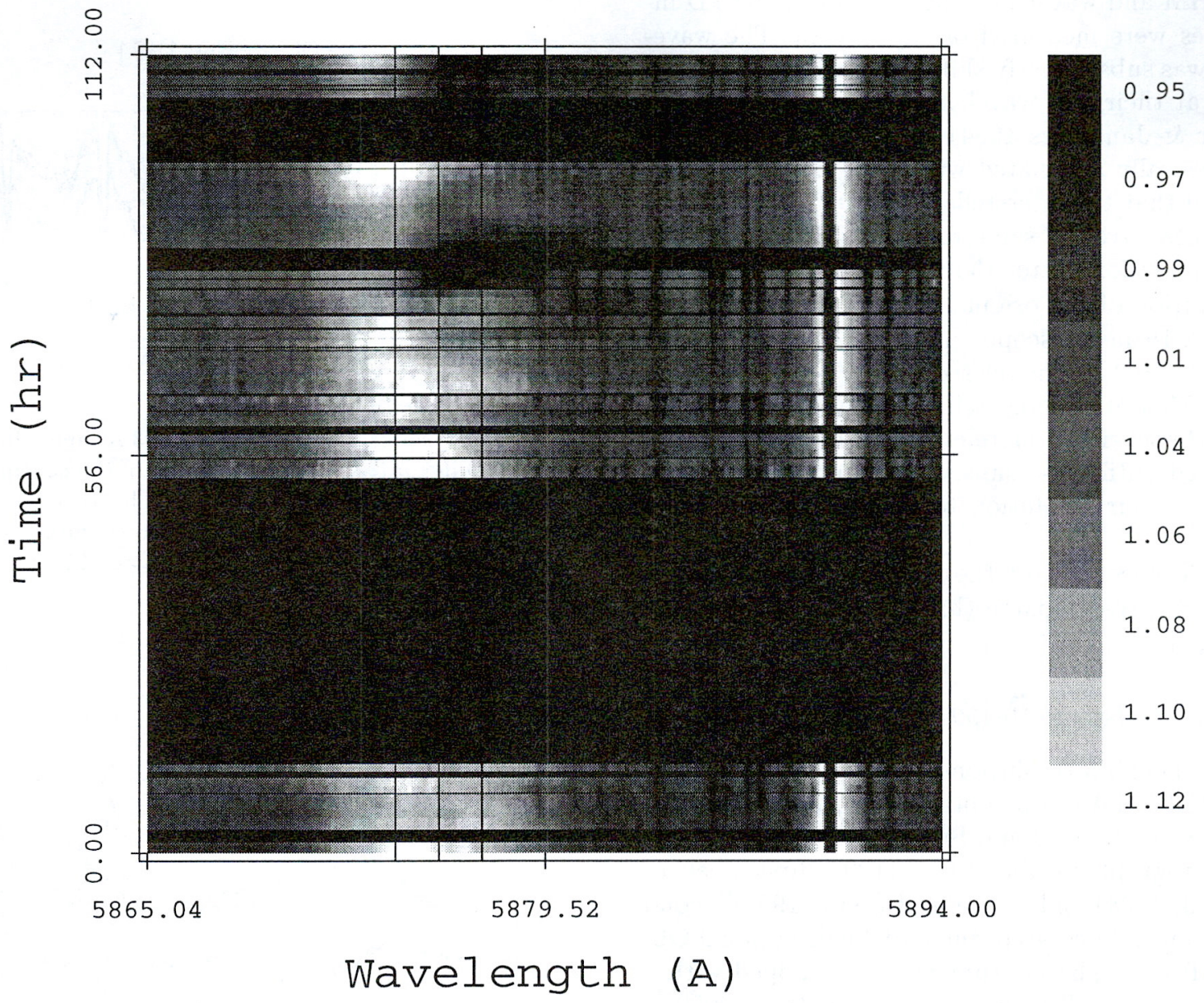

Fig. 1. Dynamic spectrum of the HeI $5875.63 \AA$ line, obtained during the MUSICOS 1992 campaign. The three thin vertical lines correspond to the rest wavelength of the line and the corresponding maximum rotational broadening at $\pm v \sin i(v \sin i=80 \pm 5$ $\mathrm{km} \mathrm{s}^{-1}$; Böhm \& Catala 1993b)

stellar spectrum. The resulting spectrum was identical to that obtained by the standard procedure to within $0.1 \%$.

\subsubsection{La Palma $4.2 \mathrm{~m}$ William Herschel telescope}

This telescope was used with the Utrecht echelle spectrograph and a $1280 \times 1180 \mathrm{EEV}$ CCD.

The data were reduced with the same software as the UH spectra (Sect. 2.1.1). A neutral density filter, used during the flatfield exposures, caused an important fringe pattern in the flatfield-spectra with an amplitude of the order of $3-4 \%$. These fringes do not exist in the stellar exposures and generate a major problem in the flat-fielding procedure. We used the following, empirically established method to "clean" the flatfield: on the optimally extracted flatfield we applied a 3 pixel mean filter followed by a 3 pixel median filter iteratively for 10 times. Thereafter we divided the optimally extracted monodimensional stellar spectrum by the corresponding filtered flatfield and pro- ceeded to a final application of a 3 pixel mean and a 3 pixel median filter. The final flat-fielded spectrum no longer shows the presence of fringes, but its $\mathrm{S} / \mathrm{N}$ ratio does certainly does not correspond to the achievable maximum since the pixel-to-pixel variations have only partly been taken into account. The final resolution was diminished by this "flatfielding" procedure from $R=40000$ to $R=31000$.

\subsubsection{Subsequent treatment}

The normalization was performed in a homogeneous way for all spectra recorded during the campaign. We used at maximum a polynomal of degree 4 to fit the continuum, ensuring always a sufficiently high number of fitting points shortward and longward of the HeI5876 $\AA$ line. Special care was taken not to affect the sometimes extended wings of the line. Cosmic ray events were manually eliminated from all spectra. 
The FWHM and wavelength position of the NaID interstellar lines were measured on all spectra. The wavelength scale was subsequently shifted so that the interstellar lines fall at their rest wavelengths: it has been shown (Finkenzeller \& Jankovics 1984) that the Herbig Ae/Be stars are physically associated with their parent molecular clouds and that the interstellar Na I D $(5889.95 \AA$ and $5895.92 \AA$ ) lines have the same radial velocity as the star itself with a precision better than about $\pm 5 \mathrm{~km} \mathrm{~s}^{-1}$.

The resolution of the original spectra was $R=31000$ at Xinglong $2.16 \mathrm{~m}$ telescope, $R=30000$ at $\mathrm{UH} 2.2 \mathrm{~m}$ telescope and OHP $1.5 \mathrm{~m}$ telescope, $R=24000$ at Kitt Peak $1.5 \mathrm{~m}$ McMath-Pierce telescope, and $R=40000$ at William Herschel $4.2 \mathrm{~m}$ telescope. The spectra were then convolved with Gaussians with appropriate widths to standardize their resolution to that of the Kitt Peak spectra.

A dynamic spectrum of the AB Aur observations of the MUSICOS core-campaign (hrs $0-112$ in Table 2) is shown in Fig. 1.

\subsection{FOE/KPNO data 1991-1994}

A large set of medium resolution HeI $5876 \AA$ observations ( $R \simeq 12000$ ) of $\mathrm{AB}$ Aur was obtained during 1991, 1992, 1993 and 1994 with the Penn State Fiber-Optic Echelle (FOE) Spectrograph at the 2.1-m (1992 [first week of the run], 1993, 1994) and coudé feed $(1991,1992$ [second week of the run]) telescopes of the Kitt Peak National Observatory (KPNO). The instrument was equipped with a T2KB CCD; the wavelength coverage was 3850-9050 $\AA$. Further details for the FOE may be found in Ramsey \& Huenemoerder (1986) and Ramsey et al. (1987). The log of the observations can be found in Table 3 .

Each observing night, in addition to object exposures, various calibration images were obtained for processing of that night's data. We used NOAO's IRAF package for all the data reduction. The average of several bias frames was subtracted from every non-bias exposure. Each stellar exposure was divided by the average of several flat field exposures to correct for the pixel-to-pixel sensitivity variations. Wavelength calibration was obtained by observing the known spectrum of a thorium-argon hollow cathode lamp.

The time series of these observations are represented in Figs. 2-5. Special attention should be given to the series of 1992, obtained less than one month before the MUSICOS 1992 campaign.

\subsection{IUE observations - Nov. 1992}

An important additional data set of the $\mathrm{MgIIh}$ and $\mathrm{k}$ UV lines was obtained one month prior to the MUSICOS campaign with IUE at a resolution of $R \simeq 15000$. The log of the observations can be seen in Table 4, the data are displayed in Fig. 6.

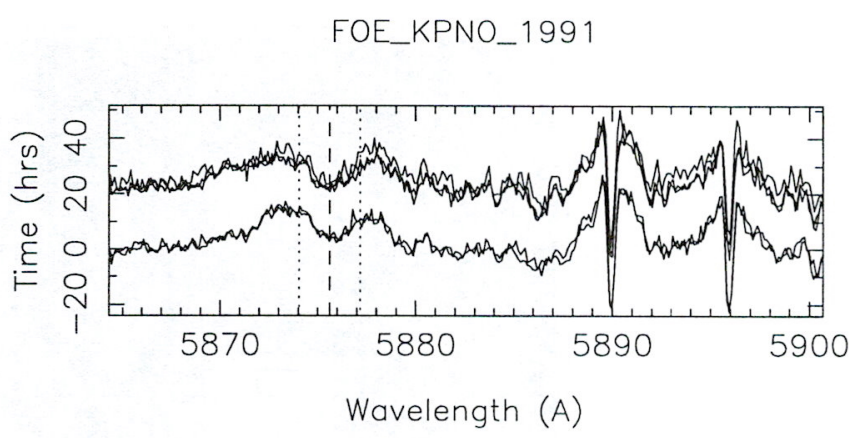

Fig. 2. Time series of the HeI5875.63 $\AA$ line obtained at FOE/KPNO in 1991. Vertical scale: $10 \%$ of the continuum correspond to $15.2 \mathrm{hrs}$ on the Time-axis. The vertical lines represent the rest wavelength and the corresponding maximum rotational broadening at $\pm v \sin i\left(v \sin i=80 \pm 5 \mathrm{~km} \mathrm{~s}^{-1}\right)$

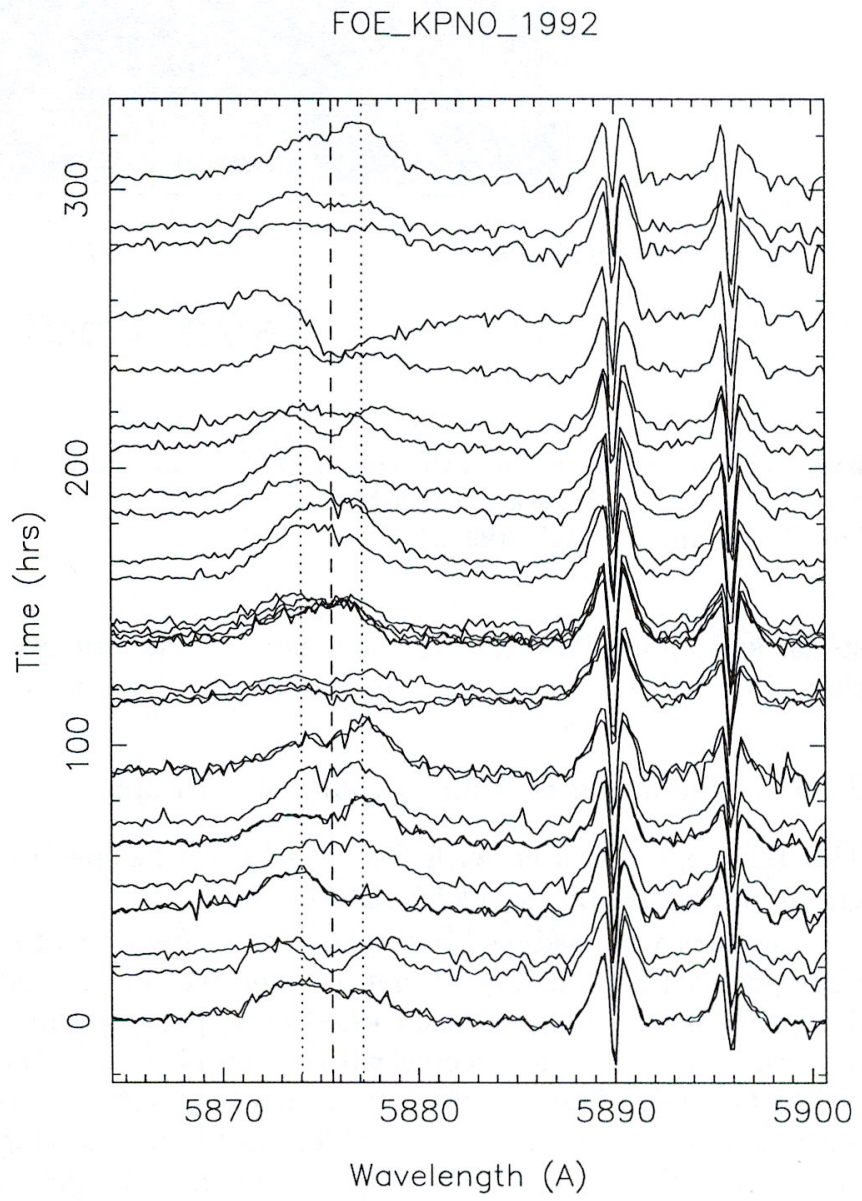

Fig. 3. FOE/KPNO data from 1992. $10 \%$ of the continuum correspond to $15.2 \mathrm{hrs}$ on the Time-axis 
Table 3. Log of the FOE/KPNO observations. Columns: (1) \# of spectrum. (2) UT date and (3) time of observations. (4) Exposure time. (5) Time difference to the first spectrum

\begin{tabular}{|c|c|c|c|c|}
\hline (1) & $\begin{array}{r}\text { UT: date } \\
(2)\end{array}$ & $\begin{array}{c}\text { UT: time } \\
\text { (beg.exp) } \\
(3)\end{array}$ & $\begin{array}{r}t_{\exp } \\
(\mathrm{s}) \\
(4) \\
\end{array}$ & $\begin{array}{r}\Delta t \\
(\mathrm{hrs}) \\
(5)\end{array}$ \\
\hline 1 & 13 Sep. 91 & 1205 & 500 & 0.00 \\
\hline 2 & & 1216 & 750 & 0.18 \\
\hline 3 & 14 Sep. 91 & 0939 & 750 & 21.57 \\
\hline 4 & & 0952 & 750 & 21.78 \\
\hline 5 & & 1228 & 481 & 24.38 \\
\hline 1 & 6 Nov. 92 & 1255 & 500 & 0.00 \\
\hline 2 & & 1306 & 150 & 0.18 \\
\hline 3 & 7 Nov. 92 & 0549 & 400 & 16.88 \\
\hline 4 & & 1300 & 400 & 24.08 \\
\hline 5 & 8 Nov. 92 & 0543 & 300 & 40.80 \\
\hline 6 & & 0550 & 300 & 40.92 \\
\hline 7 & & 1301 & 300 & 48.08 \\
\hline 8 & 9 Nov. 92 & 0541 & 300 & 64.75 \\
\hline 9 & & 0548 & 450 & 64.87 \\
\hline 10 & & 1301 & 500 & 72.10 \\
\hline 11 & 10 Nov. 92 & 0550 & 600 & 88.92 \\
\hline 12 & & 0647 & 300 & 89.87 \\
\hline 13 & 11 Nov. 92 & 0757 & 300 & 115.03 \\
\hline 14 & & 0929 & 500 & 116.55 \\
\hline 15 & & 1300 & 500 & 120.08 \\
\hline 16 & 12 Nov. 92 & 0548 & 360 & 136.88 \\
\hline 17 & & 0556 & 60 & 137.02 \\
\hline 18 & & 0752 & 300 & 138.95 \\
\hline 19 & & 0950 & 450 & 140.92 \\
\hline 20 & & 1304 & 400 & 144.15 \\
\hline 21 & 13 Nov. 92 & 0549 & 720 & 160.88 \\
\hline 22 & & 1118 & 720 & 166.38 \\
\hline 23 & 14 Nov. 92 & 0436 & 720 & 183.68 \\
\hline 24 & & 1124 & 720 & 190.48 \\
\hline 25 & 15 Nov. 92 & 0432 & 720 & 207.62 \\
\hline 26 & & 1122 & 720 & 214.45 \\
\hline 27 & 16 Nov. 92 & 0758 & 720 & 235.05 \\
\hline 28 & 17 Nov. 92 & 0356 & 600 & 255.02 \\
\hline 29 & 18 Nov. 92 & 0428 & 720 & 279.55 \\
\hline 30 & & 1043 & 600 & 285.80 \\
\hline 31 & 19 Nov. 92 & 0529 & 900 & 304.58 \\
\hline
\end{tabular}

\section{Results}

\subsection{Variability of the HeI $5876 \AA$ line}

\subsubsection{The MUSICOS 1992 data}

Figure 1 shows a dynamic spectrum of the HeI5876 $\AA$ line, constructed from the data obtained during the MUSICOS 1992 campaign on AB Aur. The time cover-

\begin{tabular}{|c|c|c|c|c|}
\hline (1) & $\begin{array}{r}\text { UT: date } \\
\text { (2) }\end{array}$ & $\begin{array}{c}\text { UT: time } \\
\text { (beg.exp) } \\
(3)\end{array}$ & $\begin{array}{r}t_{\exp } \\
(\mathrm{s}) \\
(4)\end{array}$ & $\begin{array}{r}\Delta t \\
\text { (hrs) } \\
(5)\end{array}$ \\
\hline 1 & 4 Dec. 93 & 0349 & 60 & 0.00 \\
\hline 2 & & 0351 & 360 & 0.03 \\
\hline 3 & & 0754 & 50 & 4.08 \\
\hline 4 & & 0756 & 400 & 4.12 \\
\hline 5 & & 1206 & 60 & 8.28 \\
\hline 6 & 5 Dec. 93 & 0340 & 300 & 23.85 \\
\hline 7 & & 0852 & 300 & 29.05 \\
\hline 8 & & 1216 & 240 & 32.45 \\
\hline 9 & 6 Dec. 93 & 0336 & 300 & 47.78 \\
\hline 10 & & 0734 & 240 & 51.75 \\
\hline 11 & & 1215 & 240 & 56.43 \\
\hline 12 & 7 Dec. 93 & 0332 & 300 & 71.72 \\
\hline 13 & & 0724 & 300 & 75.58 \\
\hline 14 & & 1203 & 600 & 80.23 \\
\hline 15 & 8 Dec. 93 & 0340 & 300 & 95.85 \\
\hline 16 & & 0742 & 300 & 99.88 \\
\hline 17 & & 1139 & 600 & 103.83 \\
\hline 18 & 9 Dec. 93 & 0456 & 300 & 121.12 \\
\hline 19 & & 0747 & 300 & 123.97 \\
\hline 1 & 9 Dec. 94 & 0348 & 250 & 0.00 \\
\hline 2 & & 0724 & 240 & 3.60 \\
\hline 3 & & 1124 & 240 & 7.60 \\
\hline 4 & 10 Dec. 94 & 0327 & 240 & 23.65 \\
\hline 5 & & 0723 & 240 & 27.58 \\
\hline 6 & & 1121 & 300 & 31.55 \\
\hline 7 & 11 Dec. 94 & 0402 & 900 & 48.23 \\
\hline 8 & & 0419 & 900 & 48.52 \\
\hline 9 & & 1118 & 300 & 55.50 \\
\hline 10 & 12 Dec. 94 & 0517 & 900 & 73.48 \\
\hline 11 & 13 Dec. 94 & 0337 & 300 & 95.82 \\
\hline 12 & & 0804 & 300 & 100.27 \\
\hline 13 & & 1051 & 300 & 103.05 \\
\hline 14 & 14 Dec. 94 & 0346 & 300 & 119.97 \\
\hline 15 & & 0722 & 300 & 123.57 \\
\hline 16 & & 0728 & 100 & 123.67 \\
\hline 17 & & 1101 & 300 & 127.22 \\
\hline 18 & 15 Dec. 94 & 0305 & 300 & 143.28 \\
\hline 19 & & 0644 & 300 & 146.93 \\
\hline 20 & & 1055 & 300 & 151.12 \\
\hline
\end{tabular}

age in the second half of the observation period is very good, but we experienced some weather problems in the first half, leading to an overall duty cycle of about $50 \%$. We see spectacular variations in this emission line; the HeID3 line is mostly in emission, but a deep absorption at $80 \mathrm{hrs}$ and a less deep absorption at $110 \mathrm{hrs}$ are 
FOE_KPNO_1993

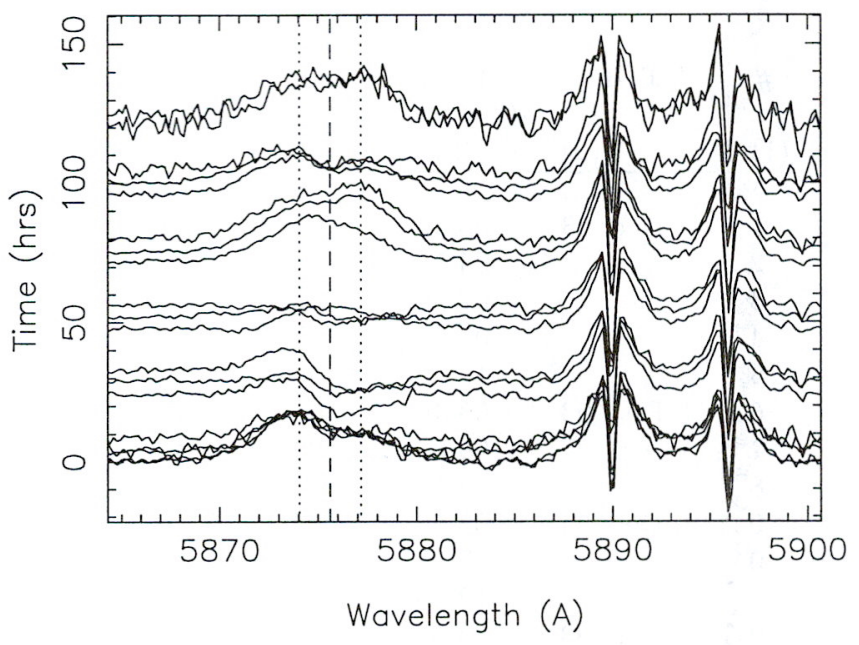

Fig. 4. FOE/KPNO data from 1993. $10 \%$ of the continuum correspond to $15.2 \mathrm{hrs}$ on the Time-axis

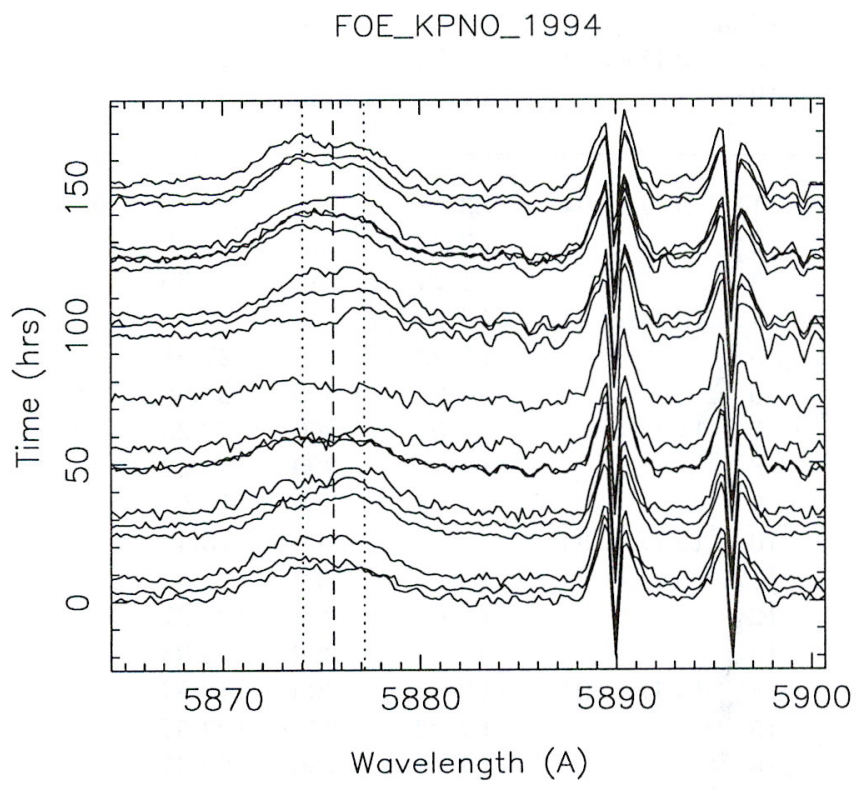

Fig. 5. FOE/KPNO data from 1994. $10 \%$ of the continuum correspond to $15.2 \mathrm{hrs}$ on the Time-axis

striking. Also, the emission seems to be somewhat blueshifted compared to the rest-wavelength of the line.

Figure 7 shows the same data set of the MUSICOS 92 campaign (Logs in Table 2), including the additional spectra obtained at UH and XL within one week after the campaign. We refer to this figure for the discussion of the profiles.

Around 0,60,65, 126 and, somewhat more intense, at $284 \mathrm{hrs}$ we observe repeatedly a similar emission-profile, which is very broad, approximately symmetric and cen-
Table 4. Log of the IUE observations of the Mg II h and $\mathrm{k}$ UV lines. Columns: (1) \# of spectrum. (2) UT date and (3) UT time of observations. (4) Exposure time. (5) Time difference to the first spectrum

\begin{tabular}{|c|c|c|c|c|}
\hline (1) & $\begin{array}{r}\text { UT: date } \\
\text { (2) }\end{array}$ & $\begin{array}{l}\text { UT: time } \\
\text { (mid.exp) } \\
(3)\end{array}$ & $\begin{array}{c}t_{\exp } \\
(\mathrm{s}) \\
(4)\end{array}$ & $\begin{array}{r}\Delta t \\
(\mathrm{hrs}) \\
(5)\end{array}$ \\
\hline 1 & 30 Oct. 92 & 2229 & 2100 & 0.00 \\
\hline 2 & & 2348 & $"$ & 1.32 \\
\hline 3 & 31 Oct. 92 & 0105 & 1800 & 2.60 \\
\hline 4 & & 0223 & $"$ & 3.90 \\
\hline 5 & & 0340 & $"$ & 5.18 \\
\hline 6 & & 0608 & 1500 & 7.65 \\
\hline 7 & & 0722 & $"$ & 8.88 \\
\hline 8 & & 0833 & $"$ & 10.07 \\
\hline 9 & & 0945 & $"$ & 11.27 \\
\hline 10 & & 1058 & 1800 & 12.48 \\
\hline 11 & & 1221 & 2100 & 13.87 \\
\hline 12 & & 1354 & $"$ & 15.42 \\
\hline 13 & & 1514 & $"$ & 16.75 \\
\hline 14 & & 1638 & $"$ & 18.15 \\
\hline 15 & & 1801 & $"$ & 19.53 \\
\hline 16 & & 2018 & $"$ & 21.82 \\
\hline 17 & & 2148 & $"$ & 23.32 \\
\hline 18 & & 2310 & $"$ & 24.68 \\
\hline 19 & 1 Nov. 92 & 0030 & $"$ & 26.02 \\
\hline 20 & & 0151 & $"$ & 27.37 \\
\hline 21 & & 0314 & 2400 & 28.75 \\
\hline 22 & & 0444 & $"$ & 30.25 \\
\hline 23 & & 0607 & $"$ & 31.63 \\
\hline 24 & & 0731 & $"$ & 33.03 \\
\hline 25 & & 0851 & 1800 & 34.37 \\
\hline 26 & & 1004 & $"$ & 35.58 \\
\hline 27 & & 1123 & 2100 & 36.90 \\
\hline 28 & & 1242 & $"$ & 38.22 \\
\hline 29 & & 1403 & $"$ & 39.57 \\
\hline 30 & & 1536 & 2400 & 41.12 \\
\hline 31 & & 1705 & $"$ & 42.60 \\
\hline 32 & & 1846 & 2100 & 44.28 \\
\hline 33 & & 2009 & $"$ & 45.67 \\
\hline 34 & & 2135 & $"$ & 47.10 \\
\hline 35 & & 2304 & $"$ & 48.58 \\
\hline 36 & 2 Nov. 92 & 0033 & 2400 & 50.07 \\
\hline 37 & & 0212 & $"$ & 51.72 \\
\hline 38 & & 0341 & $"$ & 53.20 \\
\hline
\end{tabular}

tered (or slightly blue-shifted) on the rest-wavelength of the star. This profile seems to appear the most frequently; in the following we will therefore call this profile somewhat arbitrarily the "basic"-profile of the HeID3 line. An averaged profile of spectra \# 1, 2, 14-17 and 19 (corresponding to $0.00,3.33,57.75-62.20,64.27$ ) has the following properties: an emission-peak $13 \%$ above the continuum, an equivalent width of $-750 \mathrm{~m} \AA$, a $F W H M$ of 
observations_IUE_1992

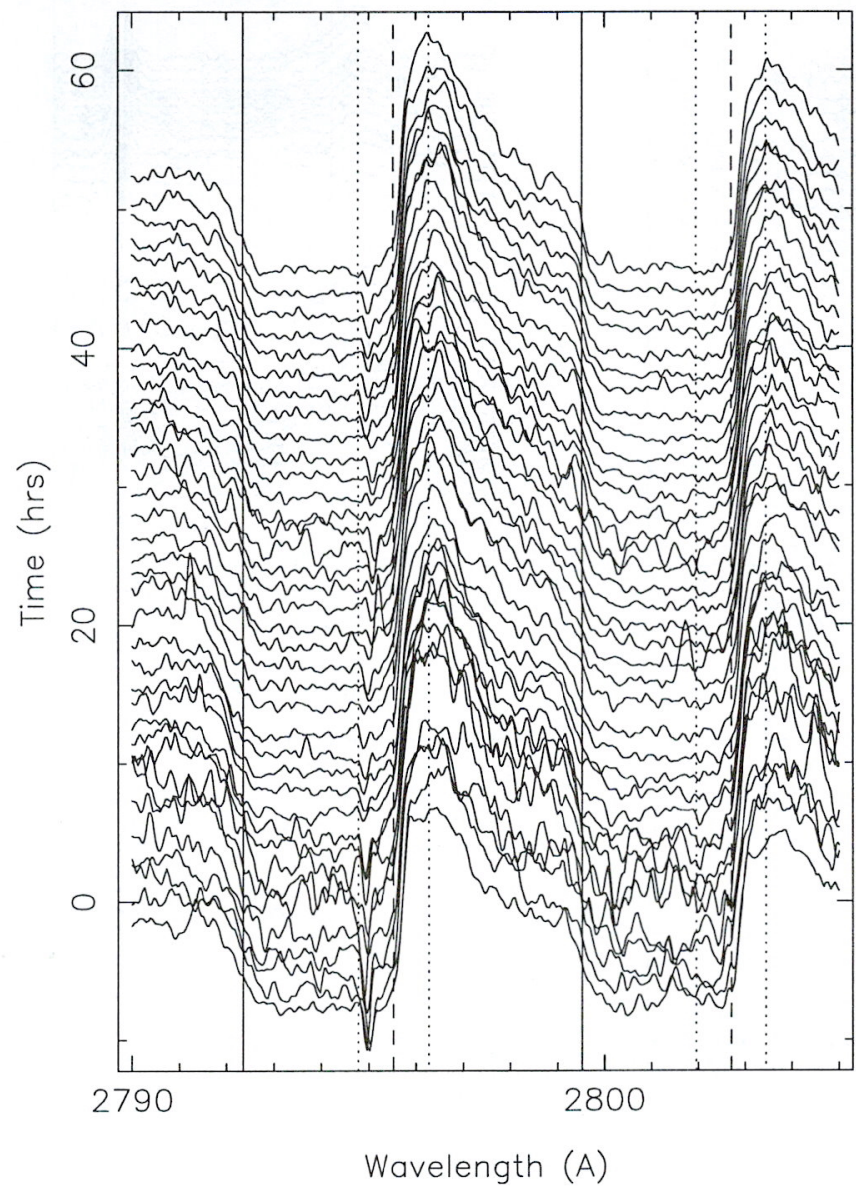

Fig. 6. Time series of the $\mathrm{MgIIh}$ and $\mathrm{k}$ UV lines observed by IUE one month prior to the campaign $(R \simeq 15000) .10 \%$ of the continuum correspond to $0.8 \mathrm{hrs}$ on the Time-axis. The two sets of vertical lines (dashed and dotted) represent the rest wavelengths of the $\mathrm{h}$ and $\mathrm{k}$ line $(2795.523 \AA$ and $2805.698 \AA$ ) and their corresponding maximum rotational broadening at $\pm v \sin i\left(v \sin i=80 \pm 5 \mathrm{~km} \mathrm{~s}^{-1}\right)$. Two additional full lines blueshifted at $340 \mathrm{~km} \mathrm{~s}^{-1}$ with respect to the central wavelengths help to locate the variations of the blue edges of both lines

$350 \mathrm{~km} \mathrm{~s}^{-1}$, an extension from -370 to $+250 \mathrm{~km} \mathrm{~s}^{-1}$, and a centroid-position of $-50 \mathrm{~km} \mathrm{~s}^{-1}$. These velocities show only the order of magnitude of the encountered velocities; the individual spectra used for this combined "typical" spectrum show easily $10-20 \%$ deviation from the values given above. This averaged profile can be seen in Fig. 8 .

The emission of spectrum \# 94 at $284 \mathrm{hrs}$ exceeded the continuum by about $17 \%$ and reached a equivalent width of $-930 \mathrm{~m} \AA$, but was perfectly centered on the restwavelength. The centroid of spectrum \# 65 at 95 hrs was shifted by $-100 \mathrm{~km} \mathrm{~s}^{-1}$.
The spectacularly broad and deep absorption component around $t \simeq 80 \mathrm{hrs}$ appeared only for about 6 hours. The spectra show then a combined profile, composed of a blueshifted emission and a redshifted absorption. The deepest absorption component of the series is seen in spectrum \# 45 at $81 \mathrm{hrs}$. Its centroid is shifted by $+100 \mathrm{~km} \mathrm{~s}^{-1}$ and it extends redwards up to $\sim 380 \mathrm{~km} \mathrm{~s}^{-1}$. The equivalent width of this component is around $320 \mathrm{~m} \AA$, and it reaches a depth of $7 \%$ below the continuum. The "blue" component is shifted by $-200 \mathrm{~km} \mathrm{~s}^{-1}$, its edge is extending bluewards up to $-540 \mathrm{~km} \mathrm{~s}^{-1}$, the equivalent width is $-260 \mathrm{~m} \AA$ and arises about $6 \%$ above the continuum. Spectrum \# 89 at 111 hrs shows even wings extending as far as $-700 \mathrm{~km} \mathrm{~s}^{-1}$ to the blue and more than $450 \mathrm{~km} \mathrm{~s}^{-1}$ to the red.

\subsubsection{FOE/KPNO data 1991-1994}

The FOE/KPNO data set from 1991 (Fig. 2) covers only 24 hrs with 5 exposures. No major variations can be detected in the HeID3 line. It can noticed that the profile resembles the profiles \# 26 at 214 hrs or \# 14 at 80 hrs in the FOE/KPNO data set from 1992 or 1993, respectively.

A very interesting $\mathrm{FOE} / \mathrm{KPNO}$ data set was obtained in 1992 (Fig. 3). The data cover 305 hrs with 31 spectra. Spectrum \# 28 at 255 hrs shows again the presence of a very deep absorption component. Its centroid is shifted by $+100 \mathrm{~km} \mathrm{~s}^{-1}$ and it extends redwards as far as $\sim 400 \mathrm{~km}$ $\mathrm{s}^{-1}$; the equivalent width of this component is $420 \mathrm{~m} \AA$ and it reaches a depth of $10 \%$ below the continuum. The "blue" component is shifted by $-220 \mathrm{~km} \mathrm{~s}^{-1}$, its edge is extending bluewards as far as $-520 \mathrm{~km} \mathrm{~s}^{-1}$, the equivalent width is $-200 \mathrm{~m} \AA$ and rises almost $6 \%$ above the continuum. The variability of the HeID3 line is again tremendeous during these observations. Nevertheless, we observe again a "basic"-profile similar to the one observed in the MUSICOS 1992 data set, but somewhat flat-topped). An average of different "basic"-profiles (\# 7, 10, 11, 12, 21 and 25 ) is shown in Fig. 8. Its properties are similar to the one from MUSICOS 1992: a well centered emission with $W_{\lambda} \simeq-650 \mathrm{~m} \AA$, extending from -290 to $+270 \mathrm{~km} \mathrm{~s}^{-1}$, with a maximum height of $10 \%$ over the continuum.

The FOE/KPNO data set from 1993 contains 19 spectra spanning over $124 \mathrm{hrs}$. Both "standard" features appear again. The averaged "basic"-profile (Fig. 8) of the similar spectra $12,13,14,18,19$ has a maximum height of $11 \%$, a blue and a red edge of -350 and $+270 \mathrm{~km} \mathrm{~s}^{-1}$, respectively; the equivalent widht is $-700 \mathrm{~m} \AA$ and the centroid is shifted by only $14 \mathrm{~km} \mathrm{~s}^{-1}$. Spectrum \# 8 at $32 \mathrm{hrs}$ has an absorption component with a depth of $5 \%$ of the continuum extending redwards as far as $320 \mathrm{~km} \mathrm{~s}^{-1}$, an equivalent width of $170 \mathrm{~m} \AA$ and a centroid displacement of $+110 \mathrm{~km} \mathrm{~s}^{-1}$. The "blue" component is shifted -170 $\mathrm{km} \mathrm{s}^{-1}$ bluewards and extends as far as $-450 \mathrm{~km} \mathrm{~s}^{-1}$. Its emission reaches $5 \%$ above the continuum and has an equivalent width of $170 \mathrm{~m} \AA$. 
observations_MUSICOS_1992

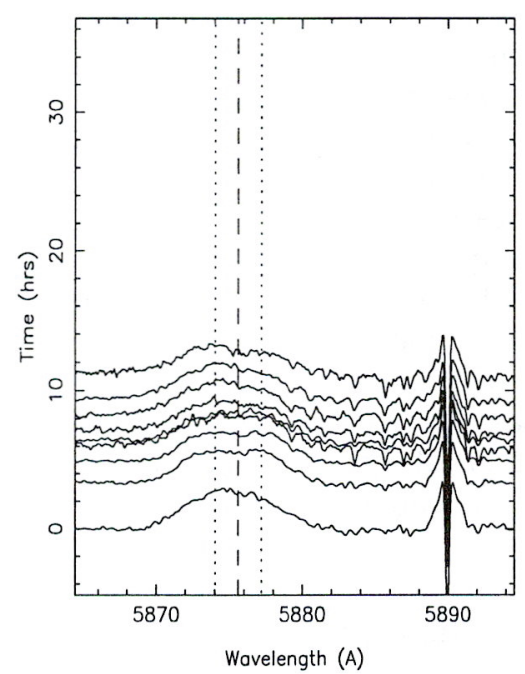

observotions_MUSICOS_1992

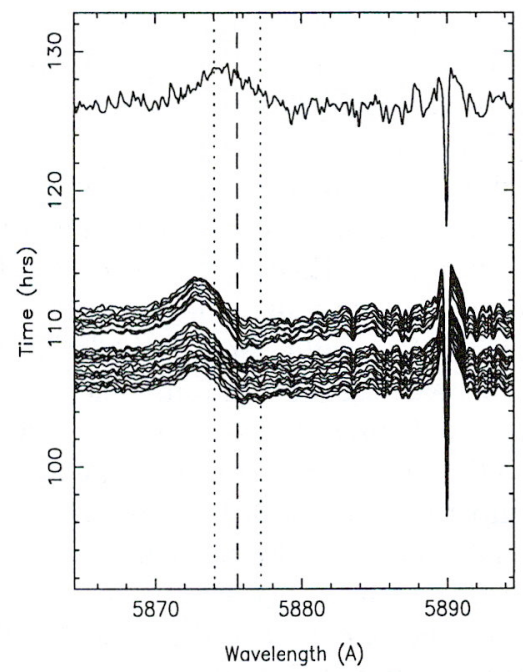

observations_MUSICOS_1992

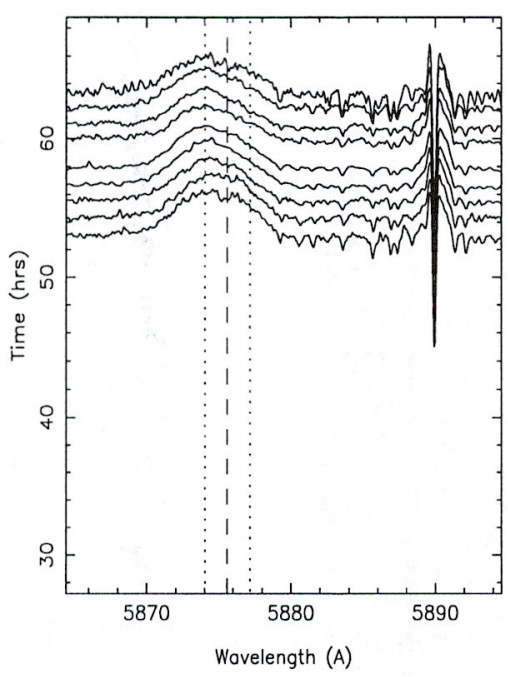

observotions_MUSICOS_1992

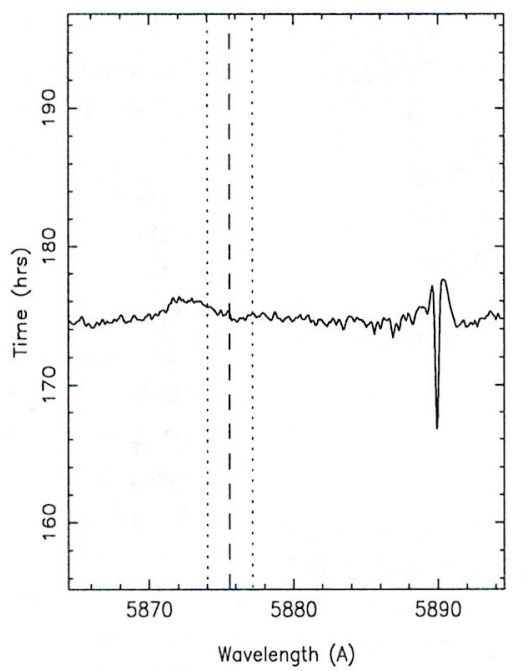

observations_MUSICOS_1992

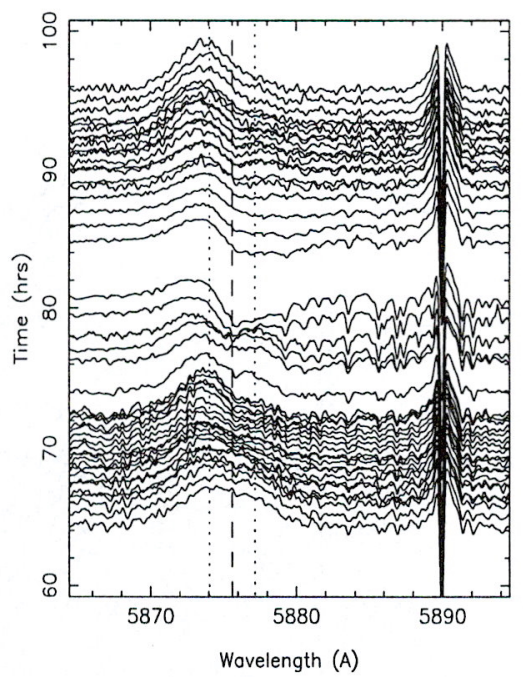

observations_MUSICOS_1992

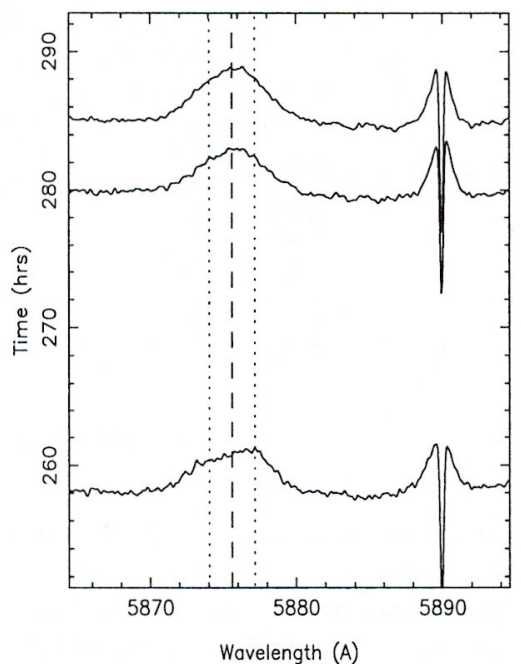

Fig. 7. Time series of the HeI5875.63 $\AA$ line, obtained during the MUSICOS 1992 campaign, containing the additional UH and XL spectra (\# 90-94). Only the excesses to the continuum (of the normalized spectra) are plotted; $10 \%$ of the continuum correspond to $2.24 \mathrm{hrs}$ on the time-axis. The individual panels correspond to blocks of $32 \mathrm{hrs}$ in the time domain [consistent with the stellar rotation period $\left(P_{\text {rot }} \simeq 32 \mathrm{hrs}\right.$ )]. The vertical line corresponds to the rest wavelength of the line and the corresponding maximum rotational broadening at $\pm v \sin i\left(v \sin i=80 \pm 5 \mathrm{~km} \mathrm{~s}^{-1}\right)$. This representation shows well the different types of profiles we observed, while Fig. 1 shows better the continuous variation of the profile

The 1994 data set (Fig. 5) shows only emission components for the HeID3 line. It's basic profile is shown in Fig. 8 and has properties comparable to the MUSICOS-1992"basic"-profile. No clear absorption appears, the variations seem also very complex.

\subsection{Rotational modulation?}

We know from previous studies of ABAur that spectral lines formed in its wind, like the CaII K line or the MgII h\&k lines, are modulated with periods close to the expected rotation period. Studies by Praderie et al. (1986) and Catala et al. (1986) reveal that the Ca II K line shows a periodic behavior with a period of 32 hours, while the Mg II h\&k variations exhibit a period of 45 hours; these authors suggest that the CaIIK line, formed near the base of the wind, is modulated with the star's rotation period, while the $\mathrm{MgII}$ \&k lines, formed further out in the wind, are modulated with a longer period corresponding to the rotation period of more remote parts of the stellar envelope. In this model, the variations would be due to 
alternating fast and slow streams in the stellar wind, controlled by a magnetic field.

The variability of the $\mathrm{MgIIh \& k} P$ Cygni profile of ABAur, observed with IUE one month before the MUSICOS 1992 campaign is in agreement with a period close to $46 \mathrm{hrs}$. The detailed analysis of the blue wings of both lines shows nevertheless that a periodicity as short as $32 \mathrm{hrs}$ would not correctly match the data. However, even if the small time-basis of the data set makes any precise periodicity determination impossible, the observed behavior resembles the one from 1982 and 1984 for these lines.

A thorough time variability analysis in all the data sets of the HeID3 line in $1 \AA$ wide wavelength bins, but also for the total equivalent width of the line, was subsequently performed. We applied very different methods, but the most efficient were the "Phase Dispersion Minimization" (Jurkevich 1971; Stellingwerf 1978) and the "Information Entropy" method proposed by Cincotta et al. (1995).

Figure 9 shows the result of the "Information Entropy"-analysis applied on the total equivalent width (EW) of the line, integrated for each spectrum between $5870 \AA$ and $5882 \AA$. After rephasing the data set with a given period, the information entropy of the resulting $2 \mathrm{D}$ cloud of points has been calculated on a $3 \times 3$ grid, following Cincotta et al. (1995). A clear minimum of the entropy is detected for a period close to $32 \mathrm{hrs}$. In order to verify the physical significance of this result, we performed a consistency-check by applying the same method to random data sets, each one obtained in the following way: for all points $\left(t_{\mathrm{obs}_{i}}, E W_{i}\right)\left[t_{\mathrm{obs}}\right.$ is the time of observation, $i$ indicates a spectrum number within the time series] we replaced $E W_{i}$ by $E W_{j}$, where $j$ is randomly selected from the total number of spectra constituing the analyzed time series. This random assignment therefore removes any underlying periodicity linked to a physical phenomenon. The large number of random data sets reduces the risk of detecting coincidental periodicities. The artificial data sets reveal some relative minima, which can be attributed to the window function of the data set; however, the absence of a $32 \mathrm{hrs}$ periodicity in these artificial data sets clearly supports the physical existence of this periodicity in the original data set.

Since the different periodicity analysis methods strongly suggest the presence of a period close to $32 \mathrm{hrs}$ in those data, despite the moderate phase coverage achieved during the MUSICOS 1992 campaign, we decided to make a consistency-check of the whole HeID3 data set in view of a rotational modulation with the stars rotation period of 32 hrs.

We subtracted the corresponding "basic"-profiles from each single HeID3 spectrum of all 5 data sets and rephased the data with different assumed periods; the smoothest variations in the phase diagram corresponded again to a period around $32 \mathrm{hrs}$. Figures 10-14 show the resulting phase-diagrams. For all data except the 1991 data set we decided to split the phase diagram in individual cycles, each cycle corresponding to one stellar rotation. "Cycle n.m" indicates the $n^{\text {th }}$ rotation of $32 \mathrm{hrs}$ and the fraction (=phase) $m$; cycles are counted relatively to the first spectrum of each individual data set. In fact, the strong intrinsic variability and the limited duration of the individual phenomena make an absolute time calibration senseless.

The detailed analysis of Fig. 10, corresponding to the MUSICOS 1992 campaign data, reveals different remarkable properties that can be interpreted as clues for rotational modulation.

Spectra close to integer cycle values, e.g., spectra from cycles [0.0-0.3], [1.6-2.0], [2.0-2.3], [2.8-3.0] and 3.9 are almost completely flat. Close to cycle 2.5 we observe a deep, broad and almost symmetric absorption appearing in these relative profiles. It reappears at cycles [3.3-3.5], but weakens until cycle 5.5. The wavelength position of this absorption component seems almost centered on the rest-wavelength of the line and does not move much during its appearance of a few hours; however a slight shift of the centroid towards the red side of the line seems to appear in cycles [2.3-3.0]. Notice the enormous extended wings of the profiles. Cycles [8.0-9.0] show unvariable profiles, which, like cycles [2.8-3.0], contain some minor emission and absorption components. The relative flatness of spectra close to cycle 0.4 show that the periodical phenomenon has a typical lifetime of some 5 to 10 cycles, i.e., 5 to 10 stellar rotations. The intrinsic variations between spectra at cycle 2.0 and spectra close to cycle 3.0 should be noticed and will be discussed later.

The FOE/KPNO 1991 data set is too small to make any statistical analysis of spectral recurrence. We decided therefore to take the first spectrum of the series as a "basic"-profile, even if this might not have great significance. Only the occurence of a minor (relative) absorption during the night can be observed (Fig. 11).

Figure 12 shows the rephased FOE/KPNO 1992 data set. Clear absorptions occur at cycles $0.7,6.7$ and 7.9, minor absorptions at cycles $1.3,[3.5-3.8],[5.7-6.0]$ and 7.3 . It is interesting to observe that the absorption at cycle 0.7 disappears completely until cycle 2.7 , assuming from the preceding results that the periodicity of $32 \mathrm{hrs}$ is responsible for the variations. The slowly increasing absorption feature from cycle [5.7-6.0], 6.7, 7.9 disappears completely at cycle [8.7-9.0]. This could again indicate the occurence of a phenomenon with a short life-time, of the order of several stellar rotations. These data might also suggest the additional presence of several minor absorption features (e.g., at cycle 1.3), not related to the main phenomenon. The absorption at cycle 7.9 could be the reappearence of the absorption observed 7 cycles earlier, making the assumption that physical conditions responsible for the phenomenon were transiently inactive. 


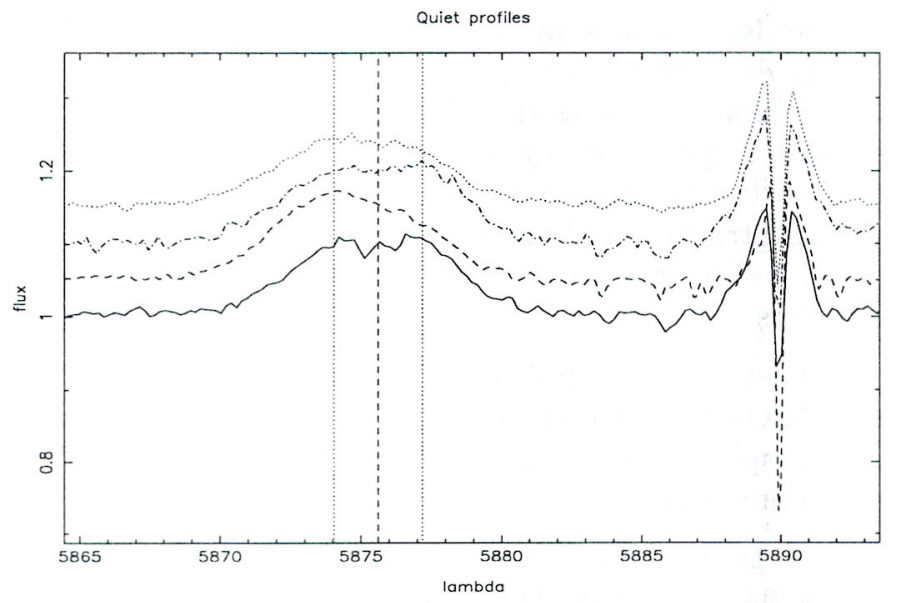

Fig. 8. "Basic" profiles from FOE/KPNO 1992 (full), MUSICOS campaign 1992 (dash), FOE/KPNO 1993 (dot-dash) and FOE/KPNO 1994 (dot)

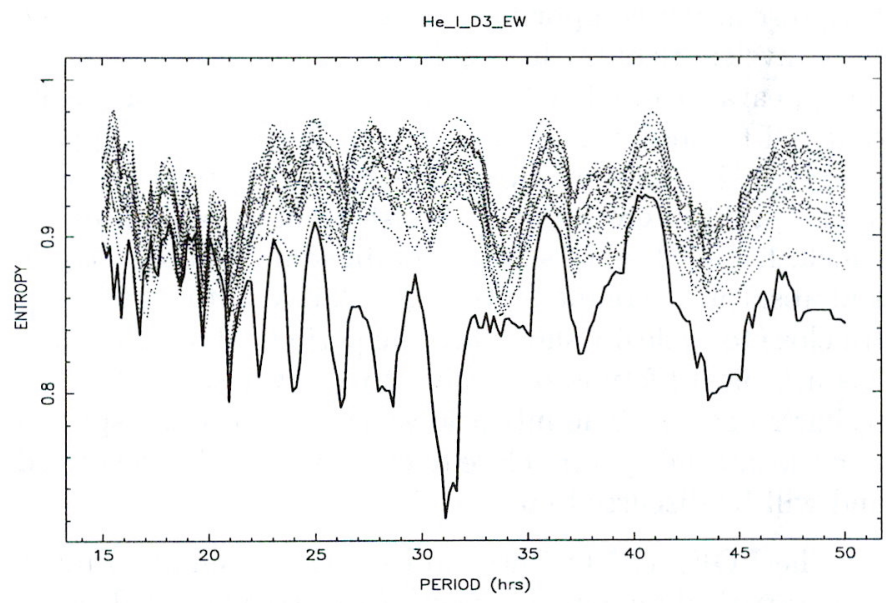

Fig. 9. "Information Entropy"-analysis of the total equivalent width (EW) of the He ID3 line time series, obtained during the MUSICOS 1992 campaign (full line). A minimum of entropy is detected for a period close to $32 \mathrm{hrs}$. The dotted lines show the same analysis, performed on artificial data sets (see text for details); the absence of any $32 \mathrm{hrs}$ periodicity in the artificial data demonstrates its physical reality in the MUSICOS 1992 data set

This idea seems to be reinforced when we take into account the FOE/KPNO 1993 spectra (Fig. 13): the deep absorption component at cycles $[0.7-0.9]$ reappear in the following cycle (1.8), but disappear completely at cycle 3.0. The absorption appearing at cycle 1.0 belongs most probably to the one previously described.

Figure 14 shows the FOE/KPNO 1994 data set treated in the same way. Only minor absorptions appear, the general situation seems very "quiet".

The general behavior of the HeID3 line in all previously discussed data set can be summarized as follows: The line is extremely variable on timescales compara- ble to one hour. Its profile is mostly in emission, almost centered on the rest-wavelength, extending red- and bluewards around $300 \mathrm{~km} \mathrm{~s}^{-1}$. This profile changes only slightly from one data set to the other, it is therefore called "basic". However, some spectra show in addition the presence of deep absorption components in the red part of the profile. These absorptions appear only for few hours and are recurrent with a periodicity close to the expected stellar rotation period of $\sim 32 \mathrm{hrs}$; their overall lifetime seem not to exceed a few stellar rotations. The rotational modulation of the line is strongly reinforced by these observations. It is interesting to notice the shape of the residual spectra once the "basic"-profiles have been subtracted: they all present rather symmetric and centered absorptions. However, in some cases the slight shifts between the centroids and the asymmetric wings of both components lead to profiles presenting a blueshifted emission and a redshifted absorption. Like the "basic"-spectra, the residual spectra have very broad wings extending redand bluewards several hundred $\mathrm{km} \mathrm{s}^{-1}$, i.e., many times the projected rotational velocity $\left(v \sin i=80 \pm 5 \mathrm{~km} \mathrm{~s}^{-1}\right.$; Böhm \& Catala 1993b). This indicates clearly that rotation can not explain by itself the broadening of the residual spectrum. Minor variations of the "basic"-profiles can most probably be attributed to intrinsic profile-variations. In some data sets the simultaneous existence of more than one recurrent phenomenon is likely. Clearly, the situation is more complex than expected on the basis of the past results on the CaII $\mathrm{K}$ and $\mathrm{Mg}$ II resonance lines.

\subsection{Variability of other spectral lines}

Interesting supplementary information is provided by the wide spectral coverage of the echelle spectra obtained with the MUSICOS spectrograph at UH on the 6th and 7th of December 1992 and the Utrecht echelle spectrograph at WHT on the 8th of December 1992. The most important non-photospheric lines were $\mathrm{H} \beta$, Fe II $5018.44 \AA$, He I $5875.61 \AA$, He I $6678.15 \AA$, O I $7773 \AA$ and $\mathrm{CaII} 8542.09 \AA$. The $\mathrm{H} \alpha$ line is saturated in some of the spectra. We adopted the following way to compare their variation throughout the 3 nights: Each spectrum of a night has been divided by the first spectrum of its corresponding night. The "ratio"-spectra were adjusted to the stellar rest-wavelength and a 3-pixel mean- and a 3-pixel median-filter were applied twice consecutively to reduce the noise. In order to increase the $\mathrm{S} / \mathrm{N}$-ratio we averaged groups of 3 or 4 consecutive spectra. Figure 15 shows the result of this treatment. All "ratio"-spectra corresponding to the different lines evolve in a very similar way: during the first night (UH Dec. 6th) most of them develop an absorption component on the red side of the "ratio"line profile; this is seen clearly for HeI $5876 \AA$, O I 7773 and $\mathrm{H} \beta$, much weaker for CaII 8542. They tend to move redwards during the night. The FeII $5018 \AA$ "ratio"-line develops also an absorption which seems centered on the 
observations_MUSICOS_1992 Period: 32.0

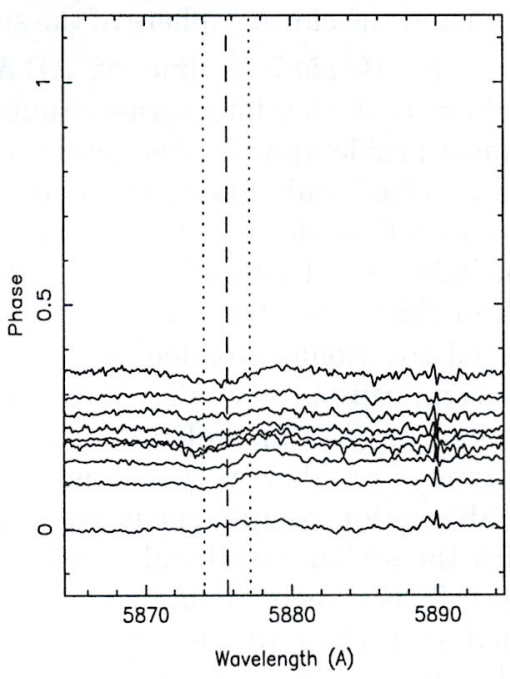

observations_MUSICOS_1992 Period: 32.0

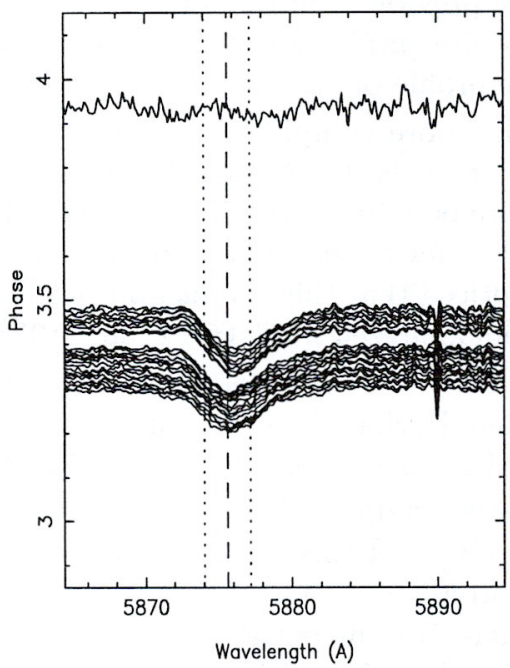

observations_MUSICOS_1992 Period: 32.0

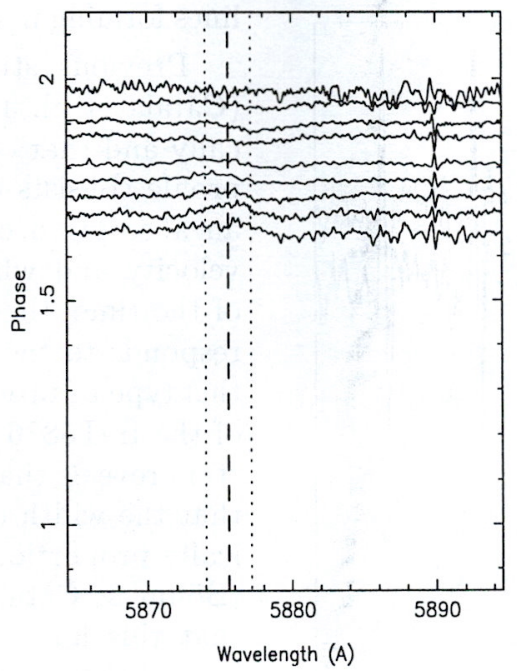

observations_MUSICOS_1992 Period: 32.0

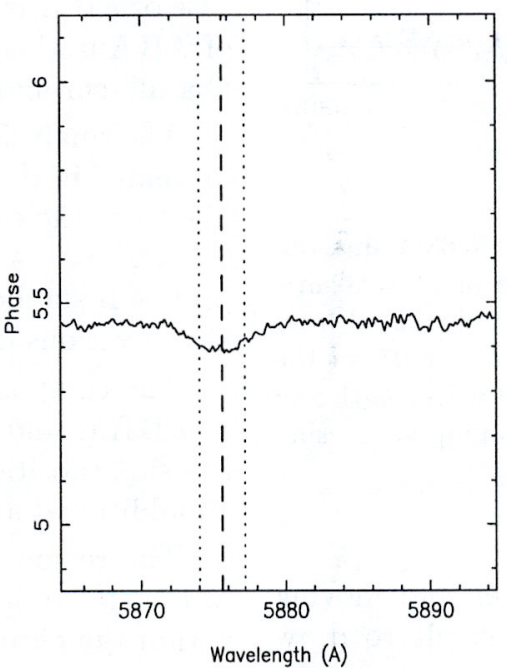

observations_MUSICOS_1992 Period: 32.0

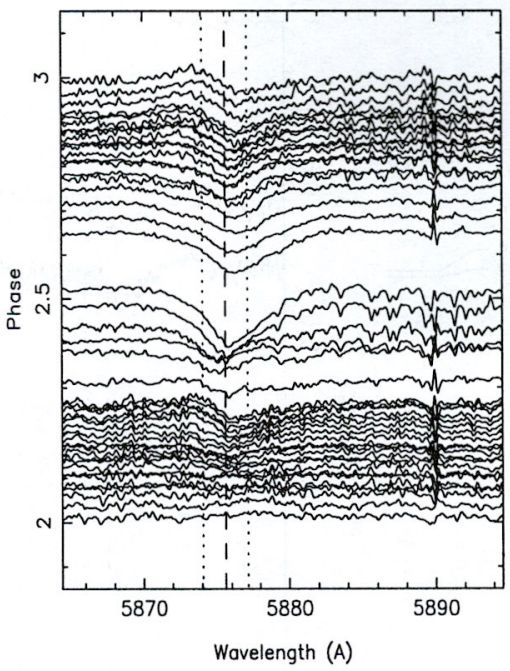

observations_MUSICOS_1992 Period: 32.0

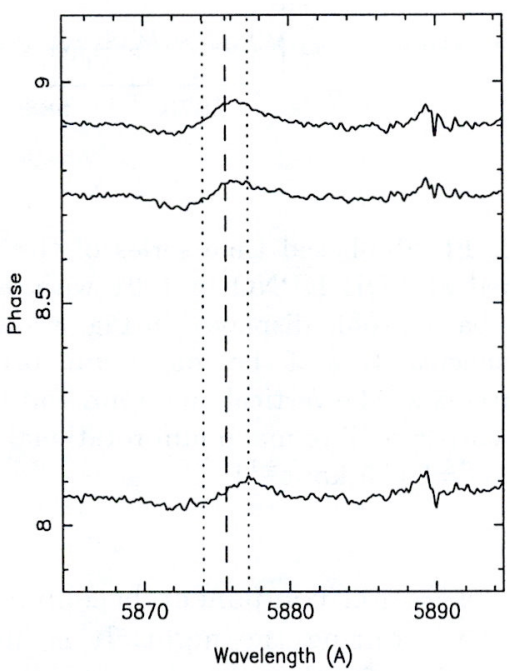

Fig. 10. Time series of the HeI5875.63 $\AA$ line, obtained during the MUSICOS 1992 campaign. The basic profile displayed in Fig. 8 has been subtracted from all spectra. $10 \%$ of the continuum correspond to 0.07 on the Phase-axis (same relative y-scale than in Fig. 7). The vertical line corresponds to the rest wavelength of the line and the corresponding maximum rotational broadening at $\pm v \sin i\left(v \sin i=80 \pm 5 \mathrm{~km} \mathrm{~s}^{-1}\right)$

rest-wavelength of the line. $\mathrm{H} \beta$ shows in addition two complex absorption components developing on the blue side of the "ratio"-line. All of them develop more or less strong blueshifted emission during this first UH night. The simultaneous development of emission during the second UH night (Dec. 7th) reinforces the idea of a covariation. This emission (in the "ratio"-profile) seems to arise more intensively in the blue part of the profile. In night 3 at WHT (Dec. 8th) all "ratio"-profiles develop absorption on the red side of the profile, some also emission on the blue side. The $\mathrm{H} \beta$ line, which, unlike the others, is formed thoughout all the stellar atmosphere, exhibits for this rea- son a somewhat more complex behavior than the other lines.

Very low-level variations at the limit of detectability are present in many photospheric lines. These variations consist in a slight change in the asymmetry of the lines, and occur with time-scales of hours. The analysis of these variations using the complete set of photospheric lines present in the echelle spectra of these nights is presented in Catala et al. (1996). Catala et al. computed residual spectra with respect to an averaged photospheric profile and plotted thereafter "ratio"-spectra of the residuals in the same way as in Fig. 15. A comparison shows that during the first night even the photospheric "ratio"-lines develop 


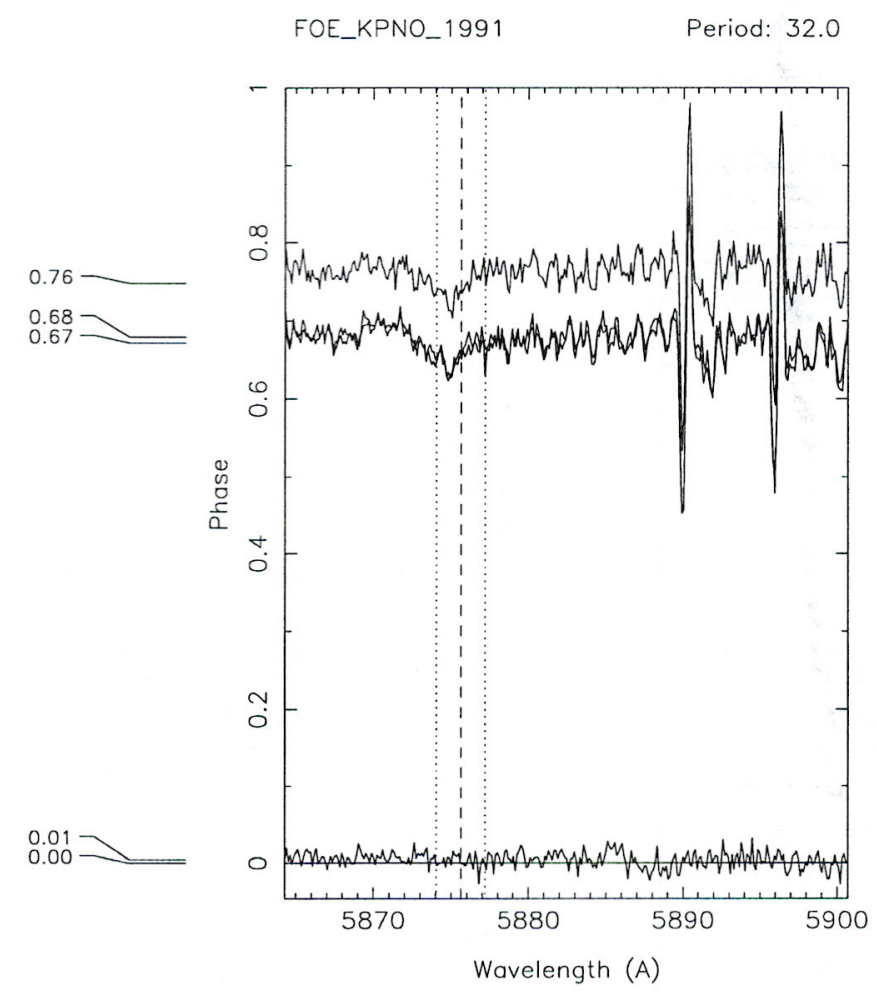

Fig. 11. Rephased time series of the HeI5875.63 $\AA$ line obtained at FOE/KPNO in 1991 with a phase of $P=32 \mathrm{hrs}$. The basic profile displayed in Fig. 8 has been subtracted from all spectra. $10 \%$ of the continuum correspond to 0.07 on the Phase-axis. The vertical lines represent the rest wavelength and the corresponding maximum rotational broadening at $\pm v \sin i$ $\left(v \sin i=80 \pm 5 \mathrm{~km} \mathrm{~s}^{-1}\right)$

an absorption component becoming deeper and moving redwards during the night. It is more difficult to draw conclusions for the second night. "Ratio"-spectra from the 3rd night at WHT show again some similarities in their evolution.

It has been shown by Böhm \& Catala (1993b) that the variable lines analyzed above are formed in different radial distances in the chromosphere/wind complex of the star. We now have direct clues that all the non-photospheric lines, but most probably also the photospheric lines, are varying simultaneously. That strongly supports the idea of a globally azimuthally structured wind and chromosphere of the star and confirms prior speculations (Böhm \& Catala 1993a; Catala et al. 1993).

\section{Discussion}

The results of the MUSICOS 1992 campaign, the IUE observations one month prior to the campaign, as well as the different data sets obtained with FOE at KPNO from 1991 to 1994 strongly reinforce the idea of a rotational modula- tion of the He ID3 line, but also of other non-photospheric lines forming in the wind and the chromosphere of the star.

Previous studies of the HeI5876 $\AA$ line of ABAur (Catala et al. 1993) show that this line varies dramatically and that one typical profile appears frequently: this profile consists of a blueshifted emission component and an absorption component whose deepest part is at zero velocity and which includes a red extended wing. Most of the time, the width of this absorption component corresponds to the expected rotational broadening. We call this type of profile the composite profile. The observation of the HeI $5876 \AA$ line in a large sample of Herbig Ae/Be stars reveals that this type of profile is very common, and that the width of the absorption component is systematically proportional with the stellar rotational broadening (Böhm \& Catala 1995). This clearly reinforces the idea that this line is formed very close to the star, in a region of the wind which is co-rotating with the stellar surface. Figure 16 gives an example of such a profile in the case of $\mathrm{AB}$ Aur. In the previous studies of the HeID3 line of $\mathrm{AB}$ Aur this composite profile was interpreted as the "basal"-chromosphere profile of this line.

The analysis of the more complete HeID3 data sets presented in this paper reveals that the most frequent profile is not the composite one, but rather the one we called "basic," i.e., a broad, almost centered, strong emission profile. It should be noticed that this profile appeared also in the various data sets analyzed in Catala et al. (1993).

The composite profile observed frequently in the past in AB Aur and in other Herbig stars is compatible with the superposition of this "basic" profile in emission and an additional absorption component.

The region of formation of this line is certainly close to the stellar surface, in a zone of increasing temperature within the chromosphere (Böhm \& Catala 1993b; Catala et al. 1993; Böhm \& Catala 1995). Taking into account all observations of this line analyzed until now, including the results presented in this paper, we conclude that the chromosphere of $\mathrm{AB}$ Aur generates mainly 2 components in the HeI $5876 \AA$ line, one "basic"-component and one rotationally modulated absorption component. The line never appears purely in absorption, which reveals the permanent existence of the "basic"-profile.

The existence of a modulation of the line with the star's rotation period provides a direct clue for an azimuthally structured atmosphere; these structures are corotating with the star and most probably are of magnetic origin. Both components of the line are formed in chromospheric regions, since the He I $5876 \AA$ line cannot be formed in the photosphere of an A0 star, but they witness different physical conditions of the chromosphere. Both components are usually seen simultaneously; in the case of the observations presented in this paper the "basic"component dominates, while in other observations of the same line (Catala et al. 1993) and in observations of other 
FOE_KPNO_1992 Period: 32.0

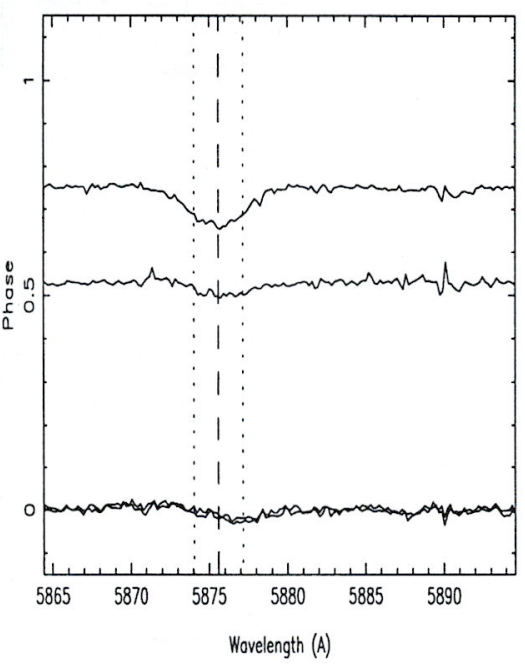

FOE_KPNO_1992 Period: 32.0

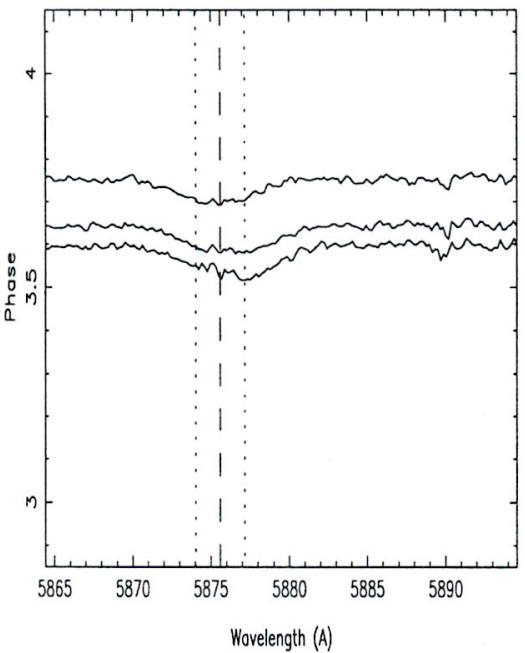

FOE_KPNO_1992 Period: 32.0

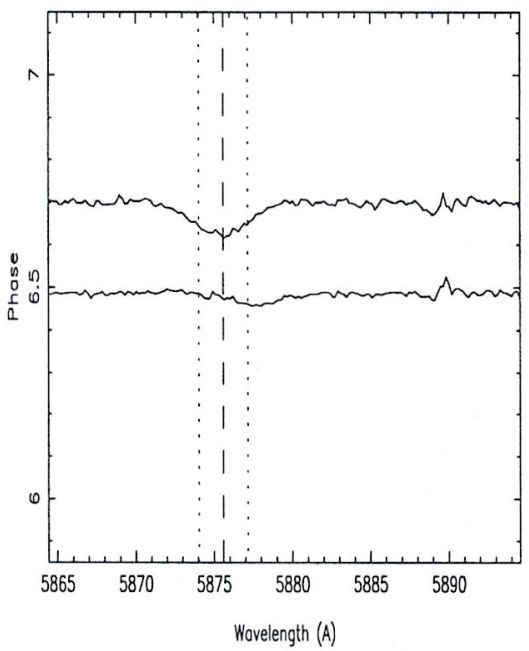

FOE_KPNO_1992 Period: 32.0

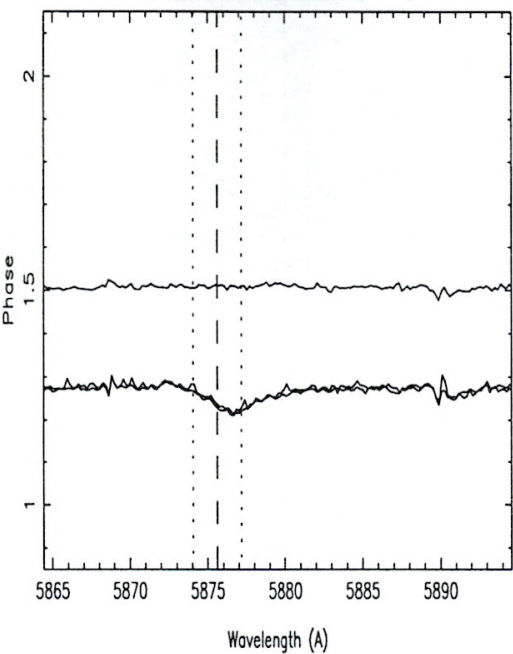

FOE_KPNO_1992 Period: 32.0

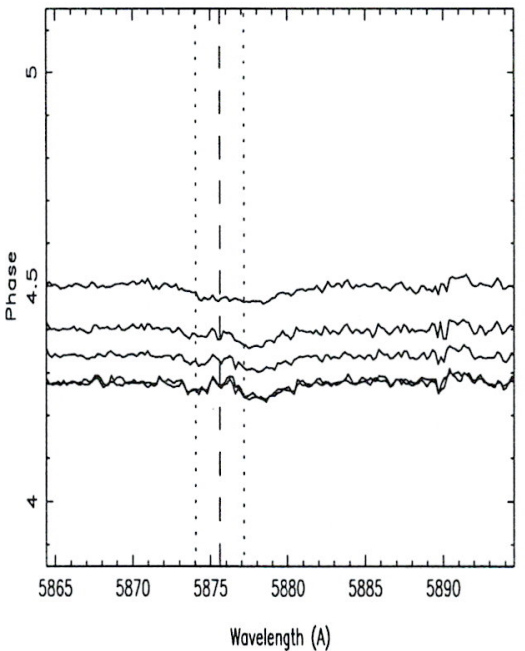

FOE_KPNO_1992 Period: 32.0

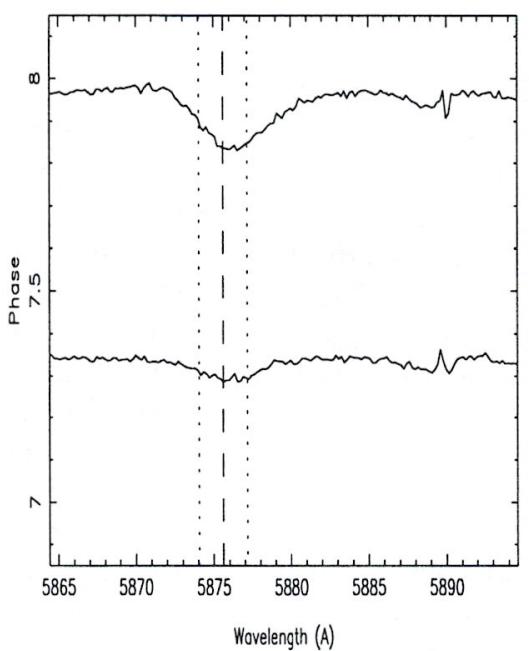

FOE_KPNO_1992 Period: 32.0

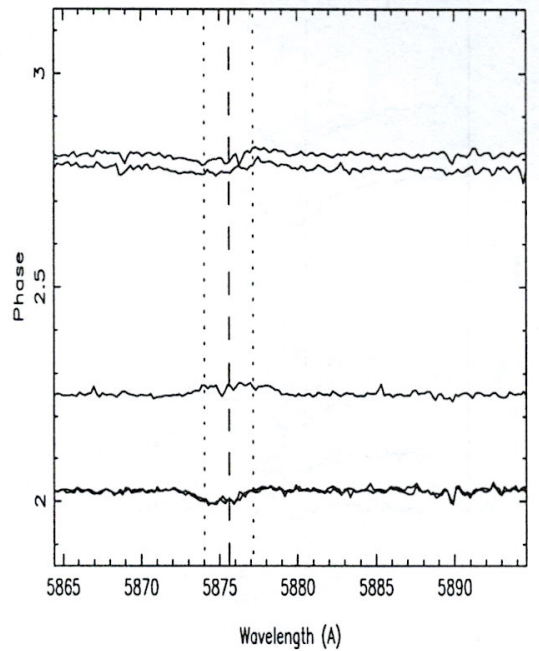

FOE KPNO_1992 Period: 32.0

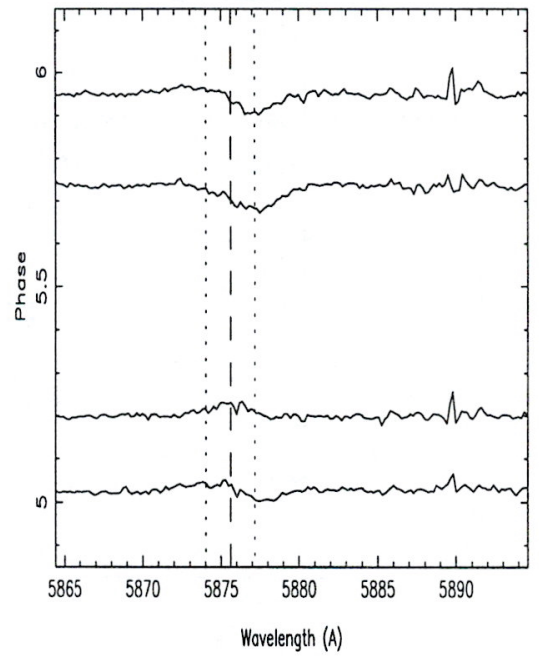

FOE_KPNO_1992 Period: 32.0

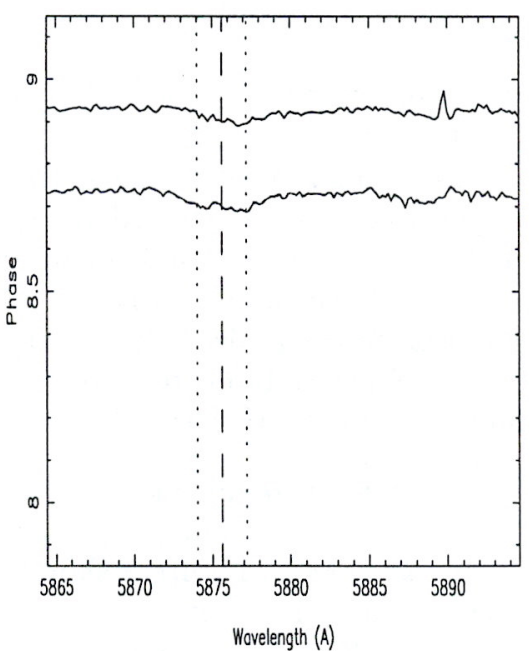

Fig. 12. Same figure as previous, but for the FOE/KPNO 1992 data 
FOE_KPNO_1993

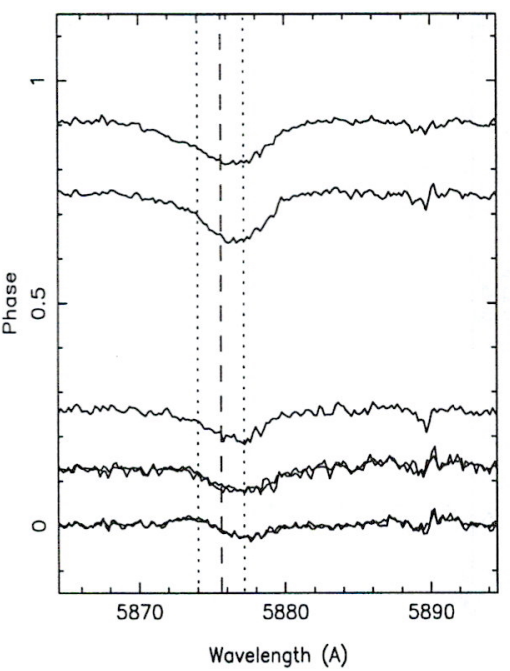

FOE_KPNO_1993

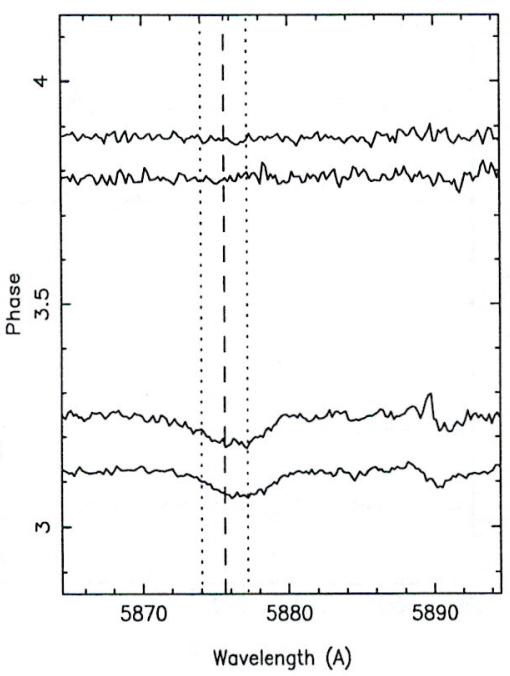

Period: 32.0

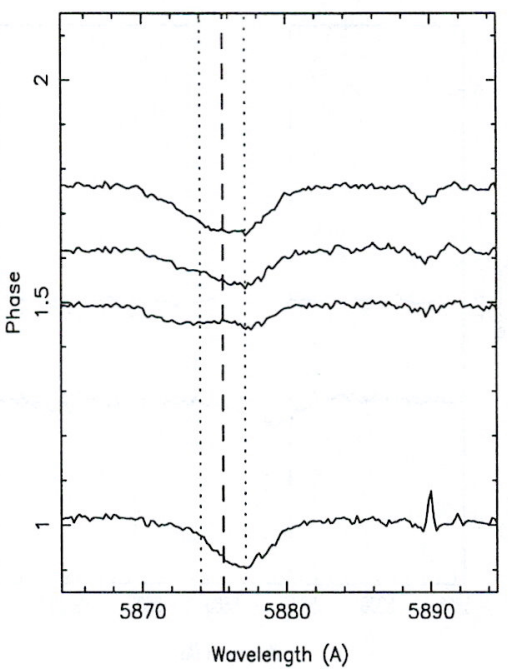

FOE_KPNO_1993

Period: 32.0

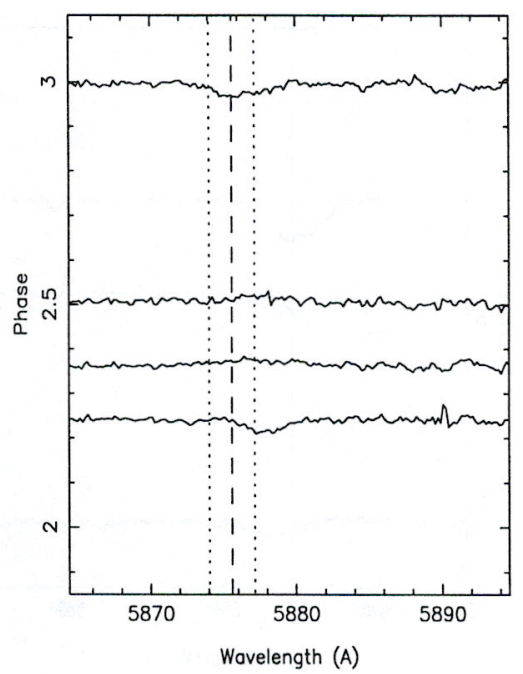

Fig. 13. Same figure as previous, but for the FOE/KPNO 1993 data

Herbig Ae/Be stars (Böhm \& Catala 1995) the absorption component seems to dominate.

Considering the very large widths of both components, it is clear that they are formed in regions of high macroturbulent motions; these motions can reach several hundreds $\mathrm{km} \mathrm{s}^{-1}$, a value consistent with previous estimates (Catala \& Kunasz 1987; Catala 1988; Catala et al. 1993). We suggest the following interpretation of the behavior of both components of the HeI $5876 \AA$ line:

- the "basic"-component is a broad and rather symmetric emission profile, approximately centered on the rest-wavelength. Figure 8 shows such profiles. It must be generated in the increasing temperature zone of the chromosphere, where the high temperature is responsible for the strong emission. Very high turbulent veloci- ties, up to several hundreds $\mathrm{km} \mathrm{s}^{-1}$, must be present in this region to account for the more or less symmetrical shape of this component.

- the absorption component is formed in hypothetical streams originating from or directed towards the stellar surface; there may be one or several of these streams. These streams are likely to be controlled by the structure of a surface magnetic field. Due to the lower densities in these magnetically confined streams, the line is formed closer to the stellar surface compared to the "basic"-component: its region of formation is therefore close to the base of the wind and chromosphere, where the chromospheric temperature is not far above the effective temperature, and is not yet high enough to generate an emission line. 

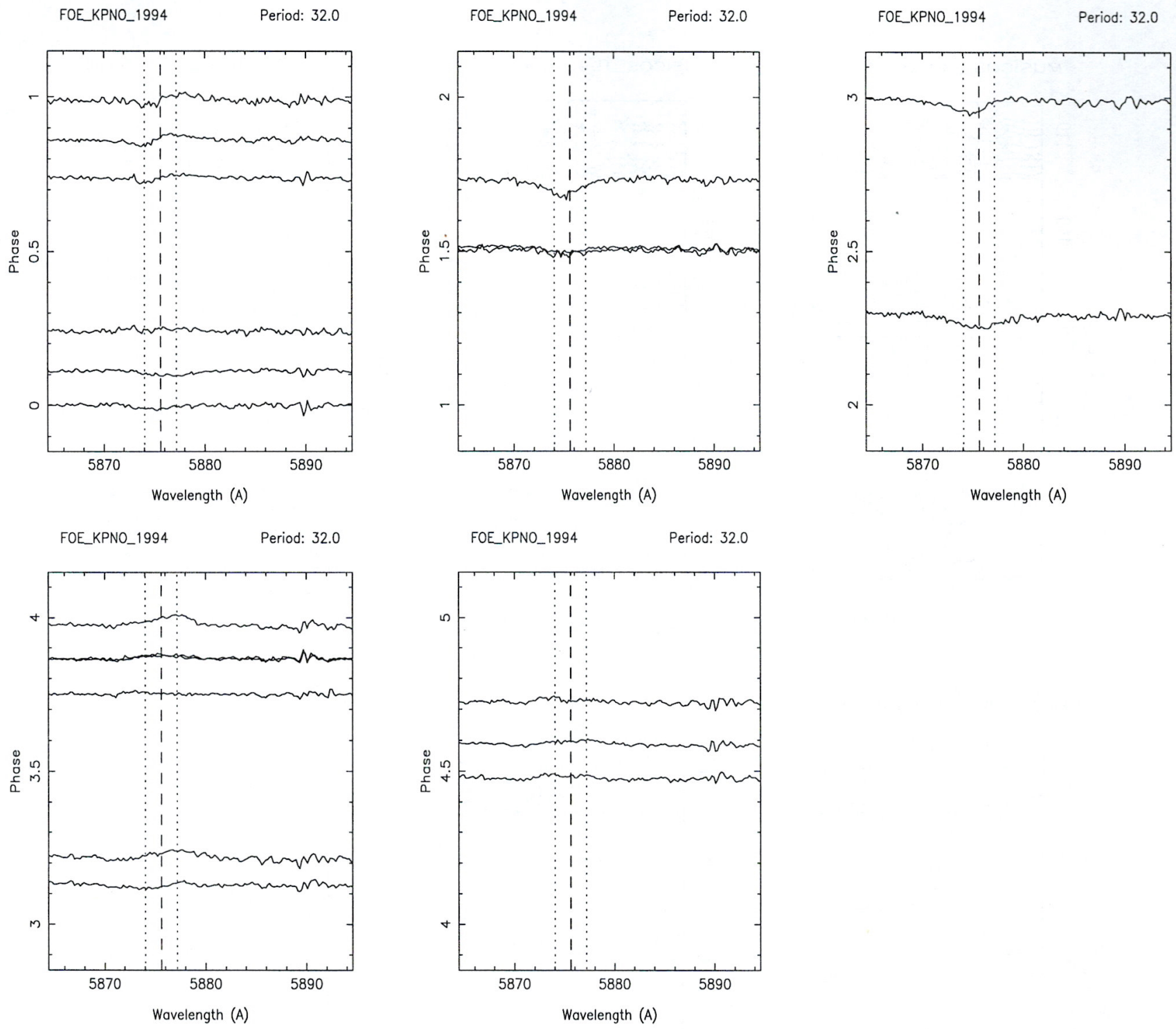

Fig. 14. Same figure as previous, but for the FOE/KPNO 1994 data

The stream structure at the origin of the behavior of the HeID3 line is also responsible for the variations of other non-photospheric lines, formed at different heights in the chromosphere. This result shows that the magnetic structure affects extended regions of the star's atmosphere, and not only those close to the surface.

If this model is valid, the very strong and recurrent absorption component observed during the MUSICOS 1992 campaign (Figs. 7 and 10) must originate from a stream component located close to the hidden pole of the star; this would explain its short appearance during cycles [2.0-3.0]. Furthermore, its lifetime seems to be of the order of a few rotation periods. This explains also the existence of longer periods of inactivity. Additional intrinsic variations of both components seem to be present, probably due to temperature, mass loss or wind velocity variations.

Three possible scenarios can be envisaged concerning the kinematics inside the streams responsible for the generation of the absorption component:

1. The streams are magnetically confined jets in which material is ejected from the star. At a certain distance the magnetic field confining these jets becomes weaker than the magnetic equipartition field and the streams are dissipated in the circumstellar medium. This would explain why the period of the modulation observed for the Mg II UV lines is longer than the stellar rotation period (see Sect. 3.2). Very high turbulent velocities would be required in these streams to explain the large widths of the absorption component. 

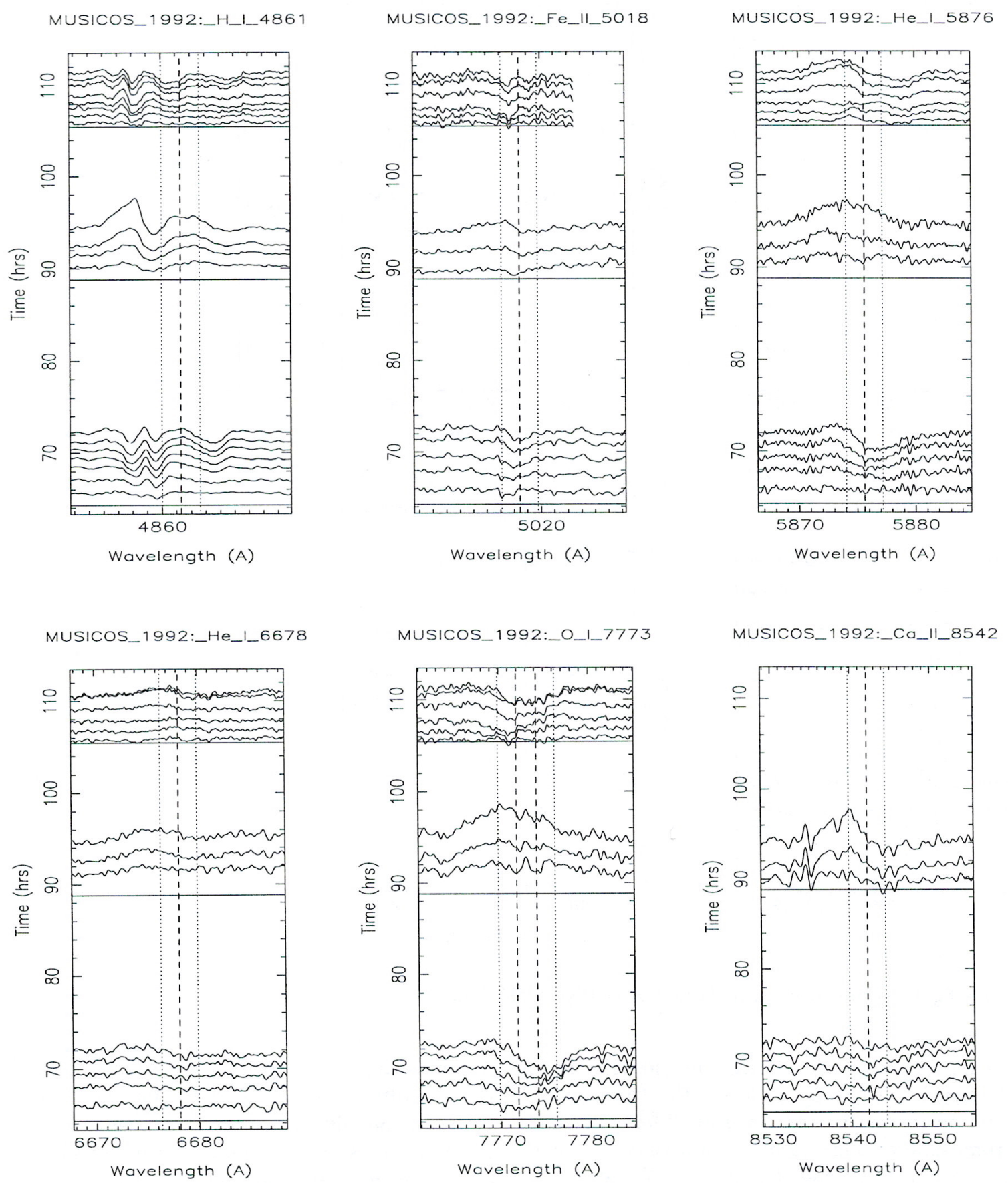

Fig. 15. Variability of other spectral lines present in the echelle spectra of UH and WHT. The time-axis is aligned with the MUSICOS 1992 campaign. The spectra cover 3 nights (2 nights UH and 1 night WHT). Each spectrum was divided by the first spectrum of the same night. These spectra contained a normalized continuum. The amplitudes $A$ (expressed in hrs) of the plotted "ratio"-profiles can be calculated by the following relation: $A=\alpha\left(\frac{F_{\lambda}}{F 1_{\lambda}}-1\right), F F_{\lambda}$ and $F 1_{\lambda}$ are the fluxes of both spectra; $\alpha$ is $14.1 \mathrm{hrs}$ for $\mathrm{H} \beta(4861.32 \AA)$ and $37.6 \mathrm{hrs}$ for the Fe II $5018.44 \AA$, HeI $5875.61 \AA$, He I $6678.15 \AA$, O I 7773 $\AA$ and Ca II $8542.09 \AA$ lines. The dashed and the dotted vertical lines correspond to the rest-wavelength and to a shift of $\pm v$ sin $i$, respectively; the dashed vertical lines in the plot of the OI 7773 line indicate the position of the two strongest single components of the O I blend at $7771.94 \AA$ and $7774.17 \AA$ 


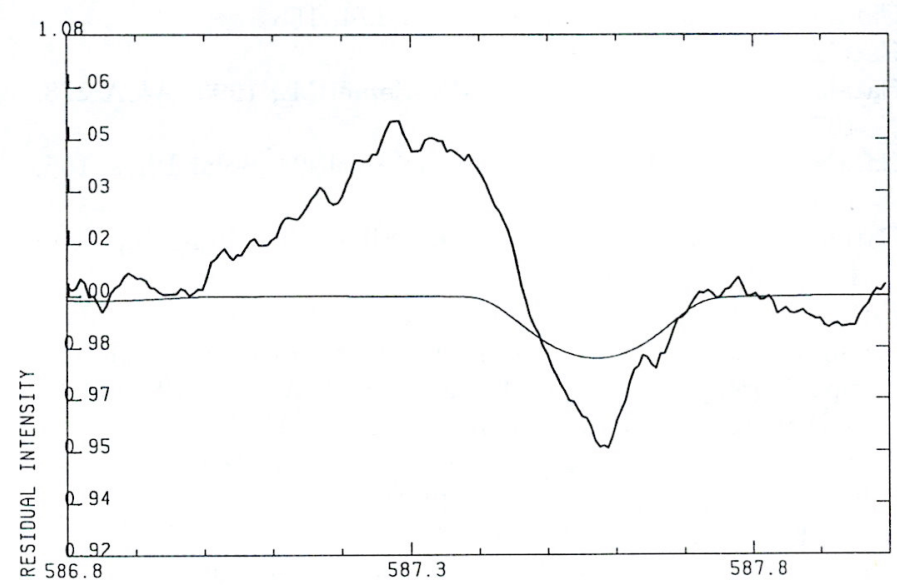

Fig. 16. Example of a typical composite profile observed in AB Aur the 16 Dec. 91 with the MUSICOS spectrograph. Superimposed (full thin line) is a synthetic photospheric spectrum, computed with the stellar parameters

2. The streams are magnetic flux tubes in which matter is falling down to the stellar surface, for instance accreted from a circumstellar disk (see e.g., Königl 1989, 1991). In this case, the flux tubes extend from a region close to the corotation radius (for AB Aur $R_{\text {corot }} \simeq 3.0 R_{*}\left[\right.$ for $M_{*}=3.2 M_{\odot}, R_{*}=2.7 R_{\odot}$ from Hillenbrand et al. 1992, $\sin i=60^{\circ}$ and $v \sin i=80 \pm 5$ $\mathrm{km} \mathrm{s}^{-1}$ from Böhm \& Catala 1993b]) the disk down to the photosphere. However, a "basic"-component is still present in this situation. This scenario seems unlikely since the presence of a significant circumstellar accretion disk around $\mathrm{AB}$ Aur and most of the other Herbig Ae/Be stars is very doubtful (Böhm \& Catala 1993b, 1994).

3. The streams are magnetic loops in which matter is ejected from the stellar surface. After moving through the loops the matter falls down again on the star. Broad absorption wings extending redwards are observed in some of the spectra described in Sect. 3.1 and could reinforce this idea. This model would not require high turbulent velocities in the loops, but rather high velocities for the material travelling along them.

The limited lifetime of the absorption component could be attributed to variations of the magnetic field responsible for the confinement of the streams.

Regarding the model, we are driven by the behavior of the other lines, e.g., MgII, to conclude that the "basic" emission component is formed over an extended region surrounding the star (but perhaps a smaller volume than produces the Mg II "P Cygni" emission feature), and that the absorption in $\mathrm{He} I$ is produced in projection against the disk (as is the MgII absorption). It is not easy to understand why most of the variations in the $\mathrm{Mg}$ II line are occuring on the blue edge of the absorption component (at velocities higher than $-340 \mathrm{~km} \mathrm{~s}^{-1}$ ), while the main variations in the HeID3 line are observed close to the rest-wavelength of the line or even slightly redshifted. It is possible that some cool material in the wind gets heated to high temperatures and then starts to fall back onto the star, while cooler gas continues to flow outward. There could be few loops, the tops of which get hot enough to produce X ray emission (Zinnecker \& Preibisch 1994), and in the bottoms of which one forms lines like He ID3, while $\mathrm{Mg}$ II and other wind lines originate in field free or open field regions.

\section{Conclusion}

A detailed analysis of AB Aur's HeI5876 $\AA$ line obtained during the MUSICOS 1992 campaign and of additional data sets yielded the following main results:

1. The strong variability of the HeI5876 $\AA$ line is confirmed. Spectacular variations within a short timescale are observed repeatedly. In certain periods of observations, the variability is much lower than in others, i.e., the star is in a "quiet" phase. In this case, the line has a broad and almost centered emission profile.

2 . The main variations are most probably due to rotational modulation of the line with a period corresponding to the star's rotation period, i.e., $P_{\text {rot }} \sim 32 \mathrm{hrs}$. A qualitative model, involving magnetically controlled streams, is compatible with the observations.

3. The stream structure observed during the MUSICOS 1992 campaign has a dominant component close to the hidden pole of the star. The lifetime of this structure is relatively short, of the order of a few rotation periods.

4. This stream structure originating from the stellar surface affects extended regions of the atmosphere, as witnessed by the co-variation of various lines probing different heights in the atmosphere.

However, due to the incomplete phase coverage and therefore the lack of continuous data in the MUSICOS 1992 campaign we are not able to prove unambiguously the hypothesis of rotational modulation and to disentangle the role of the different components of the line.

The only way to understand completely the complex behavior of AB Aur's spectral variability would be to monitor continuously a sample of spectral lines formed in different regions of the stellar atmosphere during several rotation periods. This approach would give us access to an unambiguous determination of the three-dimensional structure of the stellar atmosphere and of the star's surface.

These results confirm the presence of a structured atmosphere, with an origin of the structure close to the stellar surface at the base of the chromosphere/wind complex. Nevertheless, the origin of the structure remains unexplained and the existence of a surface magnetic field responsible for this phenomenon has still to be established. 
If confirmed, the paradoxical existence of a magnetic field in a star in radiative contraction towards the main sequence will have to be explained.

Acknowledgements. We thank the staffs of all telescopes involved in the the MUSICOS 1992 campaign for their efficient collaboration. Special thanks are due to Jeff Hall, who obtained some of the FOE/KPNO spectra. We acknowledge financial support from the "Institut des Sciences de l'Univers" (INSU/CNRS), the "Groupements de Recherche" "Magnétisme dans les étoiles de type solaire", "Structure interne des étoiles et des planètes géantes", and "Milieux circumstellaires", the Paris Observatory, the University of Hawaii, the European Space Agency, and the EEC Network of Research on "Stellar Activity". T. Böhm acknowledges with gratitude the support of the Alexander von Humboldt Foundation which funded him in 1994. Armagh Observatory is grant-aided by the Department of Education for Northern Ireland. The FOE data were obtained with support from NSF grants AST 89-19205 and AST 92-18008. Finally, we are grateful to the referee, F. Palla, for fruitful comments on the presentation of our results.

\section{References}

Baudrand B., Böhm T., 1992, A\&A 259, 711

Böhm T., Catala C., 1993a, in Inside the Stars, IAU Coll. 137, Vienne (1992), A.S.P. Conf. Ser., Vol. 40, 665

Böhm T., Catala C., 1993b, A\&AS 101, 629

Böhm T., Catala C., 1994, A\&A 250, 167

Böhm T., Catala C., 1995, A\&A 301, 155

Catala C., Felenbok P., Czarny J., Talavera A., Boesgaard A.M., 1986, ApJ 308, 791
Catala C., Kunasz P.B., 1987, A\&A 174, 158

Catala C., 1988, A\&A 193, 222

Catala C., Böhm T., Donati J.-F., Semel M., 1993, A\&A 278, 187

Catala C., Böhm T., Donati J.-F., et al., 1994, Solar Phys. 155, 185

Catala C., Böhm T., Donati J.-F., Simon T., Jiang S., Zhao F., 1996, A\&A (in press)

Cincotta P.M., Méndez M., Núñez J.A., 1995, ApJ 449, 231

Felenbok P., Guérin J., 1988, in The Impact of Very High S/N Spectroscopy on Stellar Physics. In: Cayrel de Strobel G. and Spite M. (eds.), UAI Symp. 132, p. 31

Finkenzeller U., Jankovics I., 1994, A\&AS 57, 285

Gilliland R.L., 1986, ApJ 300, 339

Horne K., 1986, PASP 98, 609

Hillenbrand L.A., Strom S.E., Vrba F.J. Keene J., 1992, ApJ 397,613

Iben I., 1965, ApJ 141, 993

Jurkevich I., 1971, Ap\&SS 13, 154

Königl A., 1989, Accretion Disks and Magnetic Fields in Astrophysics. In: Belvedere G. (ed.). Kluwer Academic Pub., Dordrecht, p. 165

Königl A., 1991, ApJ 370, L39

Praderie F., Simon T., Catala C., Boesgaard A.M., 1986, ApJ 303,311

Ramsey L.W., Huenemoerder D.P., 1986, Proc. SPIE 621, 282

Ramsey L.W., Huenemoerder D.P., Buzasi D.L., Barden S.C., 1987, In "Cool Stars, Stellar Systems, and the Sun". In: Linsky J.L. \& Stencel R.E. (eds.). Berlin: Springer, p. 515 Stellingwerf R.F., 1978, ApJ 224, 953

Zinnecker H., Preibisch Th., 1994, A\&A 292, 152 